\title{
Competition between Electronic and Magnonic Spin Currents in Metallic Antiferromagnets
}

\author{
Yan Wen, Fengjun Zhuo, Yuelei Zhao, Peng Li, Qiang Zhang, Aurélien Manchon, ${ }^{*}$ and \\ Xi-xiang Zhang $\odot^{\dagger}$ \\ King Abdullah University of Science and Technology (KAUST), Physical Science and Engineering Division (PSE), \\ Thuwal 23955, Saudi Arabia
}

(Received 17 September 2019; published 13 November 2019)

\begin{abstract}
We investigate the spin-orbit torque in a $\mathrm{Ta} / \mathrm{Ir}-\mathrm{Mn} / \mathrm{Cu} / \mathrm{Ni}-\mathrm{Fe}$ multilayer heterostructure and relate it to spin current transmission through the Ir-Mn layer. We identify several spin current transport regimes as a function of the temperature and the thickness of the Ir-Mn layer. To interpret this experiment, we develope a drift-diffusion model accounting for both electron and magnon transport in the heterostructures. This model allows us to discriminate between the contributions of electrons and magnons to the total spin current in Ir-Mn. We find that the electron-magnon spin convertance is one order of magnitude larger than the interfacial electronic spin conductance, while the magnon diffusion length is about ten times longer than the electronic spin relaxation length. This study demonstrates that magnonic spin transport dominates over electronic spin transport even in disorder metallic antiferromagnets.
\end{abstract}

DOI: 10.1103/PhysRevApplied.12.054030

\section{INTRODUCTION}

The generation and manipulation of pure spin currents lie at the core of next-generation spintronic devices used for data storage [1-3], information processing, and transmission [4]. In contrast to spin-polarized charge currents normally used in conventional spintronic devices, pure spin currents carry spin angular momentum without net charge flow and as such are considered more energy efficient [5]. Several mechanisms can generate pure spin currents, either carried by electrons or by magnons, such as the spin Hall effect (SHE) [5,6], inverse spin galvanic effect $[7,8]$, spin pumping [9-12], and spin Seebeck effect $[13,14]$. However, even though the efficient generation of spin currents has become more practical over the years, spin current transmission over long distances remains a major obstacle in practical applications. In fact, spin current transmission is limited by the spin diffusion length [15], as well as by interfacial spin memory loss and conductance mismatch (also called spin transparency) [16]. Recent progress has been achieved in nonmagnetic materials with low spin-orbit coupling such as in graphene [17], or in ferrimagnetic insulators with extremely small magnetic damping such as yttrium iron garnet (YIG) [18], demonstrating electron-mediated and magnon-mediated spin transmission over a few tens of micrometers, respectively.

\footnotetext{
*aurelien.manchon@kaust.edu.sa

†xixiang.zhang@kaust.edu.sa
}

Lately, antiferromagnetic (AFM) materials have attracted a substantial amount of attention as they can act as an efficient spin source or a spin conductor [19-26]. Enhanced magnon-mediated spin current transmission has been observed in $\mathrm{NiO}[10,14]$, as well as in $\mathrm{Fe}_{2} \mathrm{O}_{3}$, where spin transmission over distances up to $40 \mu \mathrm{m}$ was recently reported [27]. These experiments demonstrate that antiferromagnetic magnons can efficiently transmit spin current in uniaxial AFMs. The combination of long-distance transport and voltage or strain control of the magnon propagation, for example, [28-32], opens interesting perspectives for functionalized magnonic devices. Metallic AFMs present an interesting paradigm because, in principle, both electron-mediated and magnon-mediated spin currents coexist. An appealing aspect of magnonic spin current is that the conversion between electronic spin current and magnonic spin current can be very efficient. For instance, spin pumping experiments in $\mathrm{Ni}-\mathrm{Fe} / \mathrm{Fe}-\mathrm{Mn} / \mathrm{W}$ [9] and in Ni-Fe/Ir-Mn [11,33], as well as spin Seebeck measurements in YIG/Ir-Mn/Pt [13] suggest highly efficient conversion between electronic and magnonic spin current through metallic AFM. In addition, it was suggested that the magnonic spin transport through the AFM is governed by spin fluctuations, and therefore, increases close to the phase transition [34]. Although enhanced spin injection at Néel temperature and long spin current transmission have been observed, a quantitative estimation of the relative efficiency of electron-mediated and magnon-mediated spin currents is still lacking. In the present work, combining experimental detection of spin-orbit torque with theoretical 
modeling, we show that magnon-mediated spin current substantially dominates over electron-mediated spin current in a sputtered metallic AFM.

This article is organized as follows. The experimental setup and method are presented in Sec. II and the spin current measurement is discussed in Sec. III. In particular, we show that different transport regimes can be obtained by varying the sample temperature and the thickness of Ir-Mn. The theoretical interpretation is given in Sec. IV, where the drift-diffusion model is derived and compared to the experimental data. Finally, a conclusion is provided in Sec. V.

\section{EXPERIMENTAL SETUP}

To investigate the spin transmission through metallic AFM, we fabricate devices composed of heavy metal (HM)/AFM/S/ferromagnet (FM), where a heavy metal ( $\mathrm{HM}$, here Ta) is used as an electron-mediated spin current source via the SHE while the ferromagnetic layer (FM, here Ni-Fe) is used as a spin current detector. The metallic antiferromagnet (AFM, here Ir-Mn) that conveys the spin current is separated from the FM by a metallic spacer $(\mathrm{S}$, here $\mathrm{Cu}$ ) to avoid direct exchange coupling (and consequently exchange bias) between the FM layer and AFM layer. The Ir-Mn alloy with a bulk Néel temperature $T_{N}=690 \mathrm{~K}$ [25] is chosen as an AFM metal. Films of $\mathrm{Ta}(5.0) / \mathrm{Ir}_{22} \mathrm{Mn}_{78}(0-10.0) / \mathrm{Cu}(1.0) / \mathrm{Ni}_{80} \mathrm{Fe}_{20}$ (3.0)/MgO (2)/Ta (0.2) (thicknesses in nanometers) are deposited on a thermally oxidized $\mathrm{SiO}_{2} / \mathrm{Si}$ substrate by the Singulus sputter system. Throughout, we will now use Ir-Mn and $\mathrm{Ni}-\mathrm{Fe}$ to stand for $\mathrm{Ir}_{22} \mathrm{Mn}_{78}$ and $\mathrm{Ni}_{80} \mathrm{Fe}_{20}$, respectively. Since all the samples have the common structure of $\mathrm{Ta}(5.0) / \mathrm{Ir}-\mathrm{Mn}\left(t_{\mathrm{Ir}-\mathrm{Mn}}\right) / \mathrm{Cu}(1.0) / \mathrm{Ni}-\mathrm{Fe}(3.0) / \mathrm{MgO}(2) /$ $\mathrm{Ta}(0.2)$, we will refer to these samples with the thickness of Ir-Mn as "Ir-Mnt $t_{\mathrm{Ir}-\mathrm{Mn}}$," where $t_{\mathrm{Ir}-\mathrm{Mn}}$ is the AFM thickness expressed in nanometers. We pattern the samples into rectangular shapes of $8-\mu \mathrm{m}$ width and $20-\mu \mathrm{m}$ lengths using photolithography and ion beam etching. As shown in Fig. 1(a), the spin current generated by Ta flows into the Ni-Fe layer by passing through the $\mathrm{Ir}-\mathrm{Mn}$ and $\mathrm{Cu}$ layers.

The spin current absorbed by the FM layer induces a spin-orbit torque (SOT) on the FM order parameter [35]. To quantify this torque, we use the second harmonic Hall method considering both the anomalous Hall effect (AHE) and planar Hall effect (PHE), $V_{2 \omega}^{\mathrm{Hall}}=V_{2 \omega}^{\mathrm{AHE}}+V_{2 \omega}^{\mathrm{PHE}}$. We apply an ac voltage of $2.3 \mathrm{~V}$ with a frequency of $83.47 \mathrm{~Hz}$ using a locked-in amplifier. The schematic of the second harmonic method setup is shown in Fig. 1(b). The cyan arrow denotes the magnetic field rotating in the $X-Y$ plane during the measurement. In the second harmonic method, the in-plane anisotropy should be small in comparison with the external field.

\section{RESULTS}

\section{A. Spin torque measurement and determination}

To understand the basic properties of our samples, we characterize the electrical transport properties and magnetic properties of the samples under different magnetic fields over a temperature range of 20 to $300 \mathrm{~K}$. The inplane magnetic hysteresis loop [Fig. 1(c)] measured from the film of Ta (5.0)/Ir-Mn (3.0)/Cu (1.0)/Ni-Fe (3.0)/MgO $(2) / \mathrm{Ta}(0.2)$ confirms that there is no exchange bias between the FM and AFM layers. The saturated magnetization is about $600 \mathrm{emu} / \mathrm{cm}^{3}$ at $300 \mathrm{~K}$. Another effective way to demonstrate that the FM layer is not coupled to the AFM layer is to measure the planar Hall effect at a low temperature and a low magnetic field. Figure 1(d) shows that the PHE data measured at $20 \mathrm{~K}$ and with a magnetic field of 100 Oe follows the $\sin 2 \varphi$ dependence very well, which verifies that a $1.0-\mathrm{nm} \mathrm{Cu}$ layer is sufficiently thick to decouple the Ir-Mn and $\mathrm{Ni}-\mathrm{Fe}$ layers.

The principle of the second harmonic method is described as follows. When a charge current passes through the Ta layer, a net spin density $\sigma$ can accumulate in a direction transverse to the charge current. The spin current penetrates the $\mathrm{Ir}-\mathrm{Mn}$ and $\mathrm{Cu}$ layers and reaches the $\mathrm{Ni}-\mathrm{Fe}$ layer. This spin current, in general, could give two types of SOTs: dampinglike torque $\tau_{\mathrm{dl}} \sim \mathbf{m} \times(\mathbf{m} \times \boldsymbol{\sigma})$ and fieldlike torque $\tau_{\mathrm{fl}} \sim \mathbf{m} \times \boldsymbol{\sigma}$, where $\mathbf{m}$ is the magnetization unit vector of the Ni-Fe layer. Since the spin polarization is always transverse to the applied charge current, the effective field associated with the dampinglike torque is out of plane, and the effective field associated with the fieldlike torque is in plane when the magnetic moment lies in plane and parallel to the external field. These oscillating torques induced by the applied ac current cause the magnetization to oscillate both in plane and out of plane. Hence, the resistance also oscillates in the same frequency as the ac current due to AHE and PHE in Ni-Fe. Therefore, the in-plane azimuthal angle dependence of the second harmonic voltage can be divided into two components [36]

$$
\begin{aligned}
V_{\omega}= & I R_{P} \sin 2 \varphi \\
V_{2 \omega}= & I\left[\frac{H_{\mathrm{fl}}+H_{\mathrm{Oe}}}{H_{\mathrm{ex}}-H_{A}} R_{P} \cos 2 \varphi\right. \\
& \left.+\left(\frac{1}{2} \frac{H_{\mathrm{fl}}}{H_{\mathrm{ex}}-H_{K}}+c_{\mathrm{ANE}}\right) R_{A}\right] \sin \varphi
\end{aligned}
$$

where $R_{P}$ and $R_{A}$ are the coefficients of anomalous Hall effect and planar Hall effect, respectively, $H_{A}$ and $H_{K}$ are the effective in-plane and out-of-plane anisotropy fields, respectively, $V_{\omega}$ and $V_{2 \omega}$ are the first and second harmonic Hall voltages, respectively, and $H_{\mathrm{Oe}}$ is the Oersted field induced by the current. An effective anisotropy field $\left(H_{K}\right)$ of about $-5.5 \mathrm{kOe}$ is observed in the anomalous 
(a)

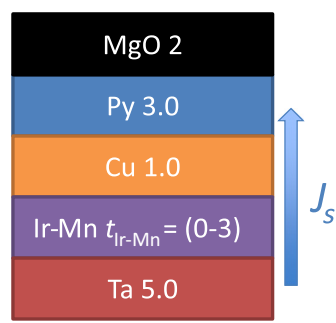

(d)

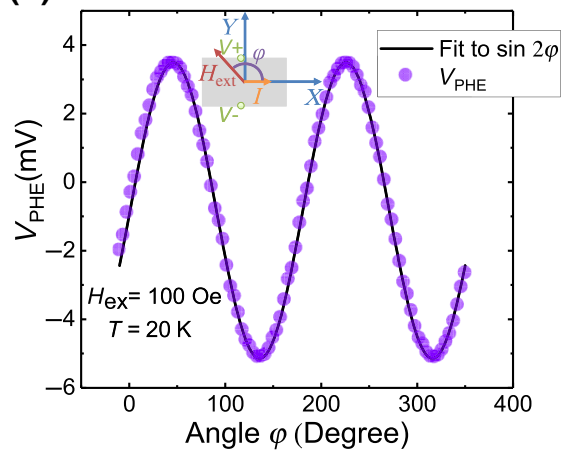

(b)

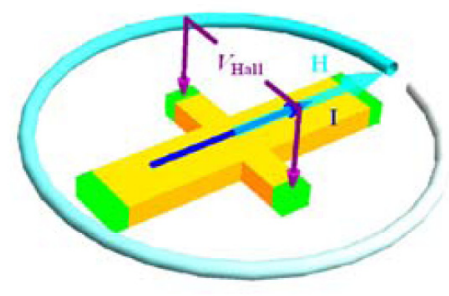

(e)

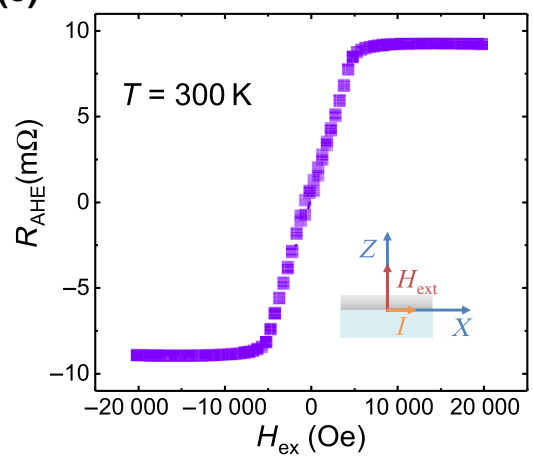

(c)

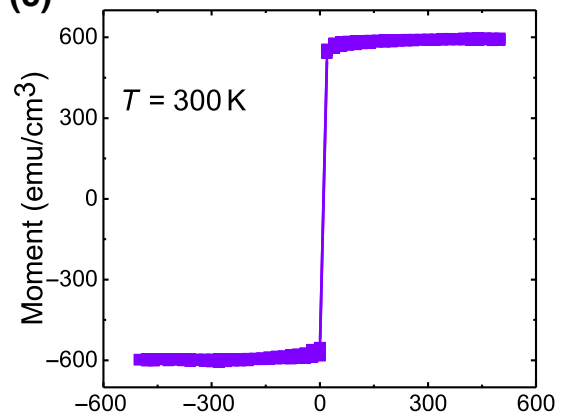

(f)

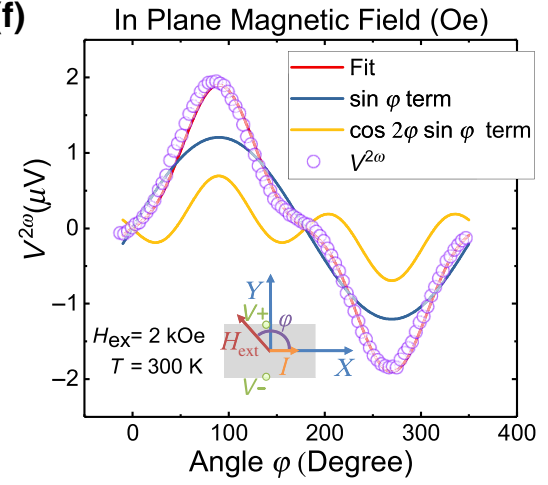

FIG. 1. (a) Illustration of the deposited film stack. (b) Schematic of second harmonic measurements. Cyan array denotes the in-plane rotated magnetic field. (c) Magnetic moment measured with the in-plane magnetic field at $300 \mathrm{~K}$. (d) Planar Hall voltage measured using a locked-in amplifier at $20 \mathrm{~K}$ with in-plane constant magnetic field $100 \mathrm{Oe}$. (e) Anomalous Hall resistance measured at $300 \mathrm{~K}$. (f) A typical second harmonic voltage measured at $300 \mathrm{~K}$ with in-plane magnetic field $2 \mathrm{kOe}$. The red line shows the fit of data. The blue and yellow lines show $\sin \varphi$ and $\sin \varphi \cos 2 \varphi$ terms, respectively. Data is captured in Ir-Mn 3.0 samples.

Hall effect results obtained at $300 \mathrm{~K}$ [see Fig. 1(e)]. Note that in addition to PHE and AHE, the thermal anomalous Nernst effect can also contribute to the measured signal with this experimental setup [37]. However, this contribution is independent of the external field, which can be described by the above equation since the current is constant during the measurement, approximately $c_{\mathrm{ANE}}$ in Eq. (2). Therefore, the thermal effect can be excluded by measuring the second harmonic voltage under several different external fields. The second harmonic voltage $V_{2 \omega}$ as a function of the azimuth angle $\varphi$ measured at $300 \mathrm{~K}$ is shown in Fig. 1(f). By fitting Eqs. (1) and (2) to the experimental data, the second harmonic signal can be separated into two contributions based on their different dependences on the azimuth angle.

We measure the second harmonic voltage in the external magnetic field ranging from 2 to $10 \mathrm{kOe}$, in which the signal is sufficiently strong and stable to obtain reliable data. To investigate how the magnetic phase transition influences the spin transport, we also vary the temperature from 20 to $300 \mathrm{~K}$. To determine the relative contributions of the fieldlike and dampinglike torques to the Hall voltage, we separate it into two contributions $V_{2 \omega}^{\mathrm{fl}}$ and $V_{2 \omega}^{\mathrm{dl}}$, corresponding to the first and second terms at the right-hand side of Eq. (2), respectively. The field dependences of $V_{2 \omega}^{\mathrm{fl}}$ and $V_{2 \omega}^{\mathrm{dl}}$ are shown in Figs. 2(a) and 2(b). The fieldlike Hall voltage, $V_{2 \omega}^{\mathrm{fl}}$, follows approximately $1 / H_{\mathrm{ex}}$ dependence [see Fig. 2(c)], which is consistent with Eq. (2). The larger the external field, the smaller the current-induced angle modulation. After taking into account the PHE voltage $V_{\text {PHE }}$, the effective field $H_{\mathrm{fl}}+H_{\mathrm{Oe}}$ can be obtained. This field, which is proportional to the slope in Fig. 2(c), is almost independent of temperature, in contrast to previous reports on fieldlike SOTs $[38,39]$. Therefore, we can ascribe this contribution to the Oersted field because it is independent of temperature. In other words, our system does not exhibit any fieldlike SOT.

The field-dependence of the dampinglike Hall voltage $V_{2 \omega}^{\mathrm{dl}}$ is displayed in Fig. 2(d). Based on Eq. (2), this signal arises from two contributions, the dampinglike SOT and the anomalous Nernst effect. In order to quantitatively separate the thermal and dampinglike field contributions, we exploit the different field dependence of $V_{2 \omega}^{\mathrm{dl}}$ as shown in Fig. 2(d). As mentioned above, the temperature gradient can only contribute to a field-independent signal. Thus, the thermal contribution is proportional to the intercept of the linear fit, while the dampinglike torque is proportional to the slope, as predicted by Eq. (2). Being consistent with previous reports on $\mathrm{Ta}$ [38-41], the dampinglike torque is mostly independent of the temperature below $300 \mathrm{~K}$. 
(a)

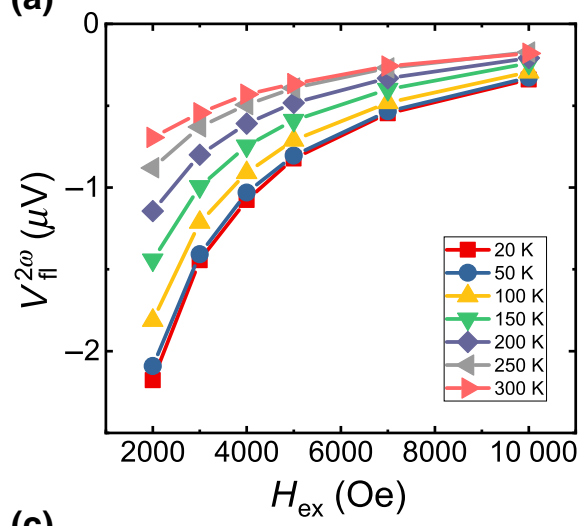

(c)

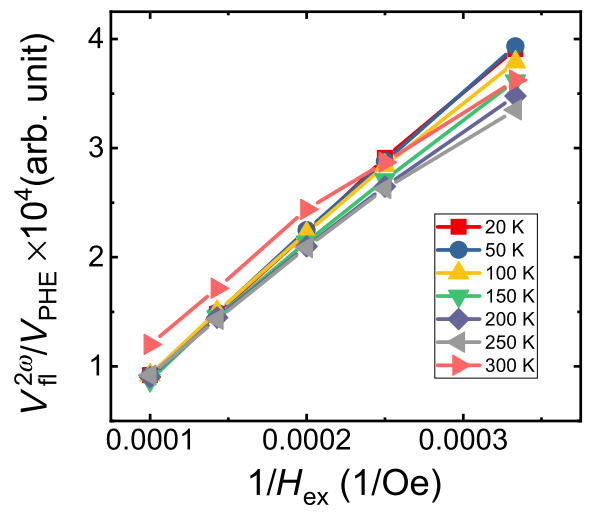

(b)

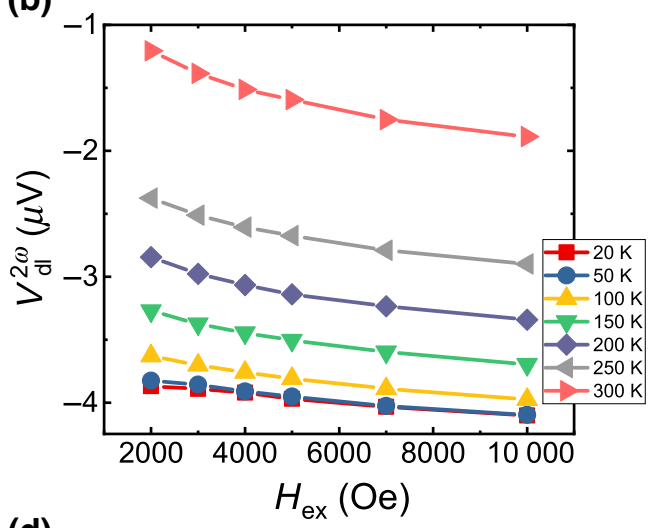

(d)

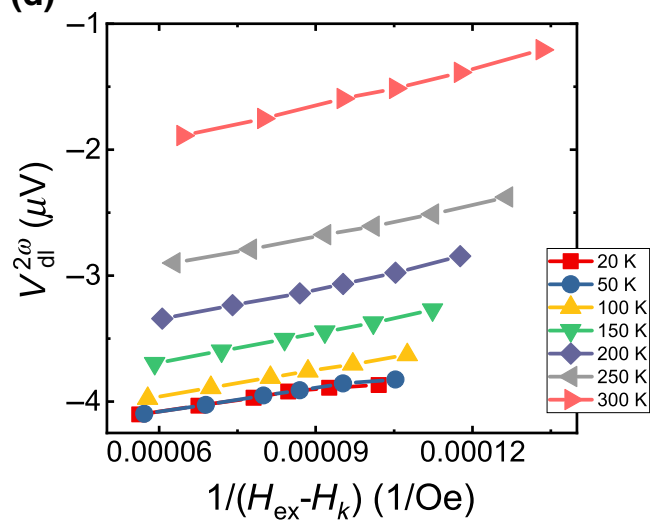

FIG. 2. (a),(b) Extracted fieldlike (a) and dampinglike (b) terms measured at different temperatures and magnetic fields. (c),(d) Linear relationship between the fieldlike (c) and dampinglike (d) terms and the reciprocal of the effective field.
To gain a deeper understanding of the intrinsic properties of the spin transport, we analyze the effective spin conductivity $\left(\sigma_{s}\right)$ calculated from the torque obtained experimentally using the definition, $\sigma_{s}=J_{s} / E=$ $H_{\mathrm{dl}} M_{s} t_{\mathrm{FM}} / E[7,8]$, where $M_{s}$ is the saturated magnetization, $t_{\mathrm{FM}}$ is the thickness of the ferromagnetic layer, and $E$ is the applied electric field in the sample. A spin conductivity, $\sigma_{s}=(\hbar / 2 e) 1.53 \times 10^{3} \Omega^{-1} \mathrm{~m}^{-1}$ is then obtained from the Ir-Mn 3.0 sample at $300 \mathrm{~K}$, in which $M_{s}=600 \mathrm{kA} / \mathrm{m}$ is extracted from Fig. 1(c) and $E=1.15 \times 10^{5} \mathrm{~V} / \mathrm{m}$.

\section{B. Temperature dependence of spin conductivity}

To determine the relative contribution of electronic and magnonic spin current to the total spin current, we vary the sample temperature and AFM thickness. Figure 3(a) displays the variation of the spin conductivity of the Ir-Mn 1.0 sample in the temperature ranging from 20 to $300 \mathrm{~K}$, which covers the phase transition temperature of the Ir-Mn 1.0 sample reported in Ref. [11]. A broad peak is observed around approximately $150-200 \mathrm{~K}$ in the curve of Fig. 3(a), which is consistent with the maximum of the enhanced magnetic damping observed by Gladii et al. [33] at the same thickness of Ir-Mn. These observations indicate that the maximum spin current transmission occurs at a temperature close to Néel temperature. To establish the relationship between the broad peak of spin conductivity and Néel temperature, we measure the exchange bias effect of the $\mathrm{Ir}-\mathrm{Mn} / \mathrm{Ni}-\mathrm{Fe}$ sample to estimate the blocking temperature, which is close to and usually lower than the Néel temperature $[14,42,43]$. The magnetic hysteresis loop measured at $50 \mathrm{~K}$ on the Ir-Mn (1)/Ni-Fe (3) film shows a nonzero exchange bias field $\left(H_{\mathrm{EB}}=43 \mathrm{Oe}\right)$, after field cooling from $300 \mathrm{~K}$ under a $2 \mathrm{kOe}$ field [inset of Fig. 3(b)]. This square loop in the inset of Fig. 3(b) suggests the Ir-Mn layer is in $\gamma$ phase [44]. From the temperature dependence of the exchange bias field and coercivity field shown in Fig. 3(b), the blocking temperature, where the exchange bias vanishes $(150$ to $200 \mathrm{~K})$, is close to the peak temperature measured from harmonic methods (150 to $200 \mathrm{~K}$ ).

This peculiar temperature dependence is consistent with previous works on spin Seebeck effect on insulators [14] or spin pumping $[9,11]$. Qualitatively, the spin current in the metallic AFM is carried by both electrons and magnons. Because of the very short electron spin relaxation length in Ir-Mn (about $0.7 \mathrm{~nm}$ at $300 \mathrm{~K}$ [23]), the temperature dependence observed in Fig. 3(a) is likely to be dominated by the magnonic spin current. As discussed in detail in Sec. IV, the magnonic transport is controlled by three parameters: the interfacial conversion between electronic spin current, called the electron-magnon spin convertance [45], the magnon conductivity in the AFM, and the magnon relaxation length. The spin convertance process is expected to display a high temperature dependence, proportional to 
(a)

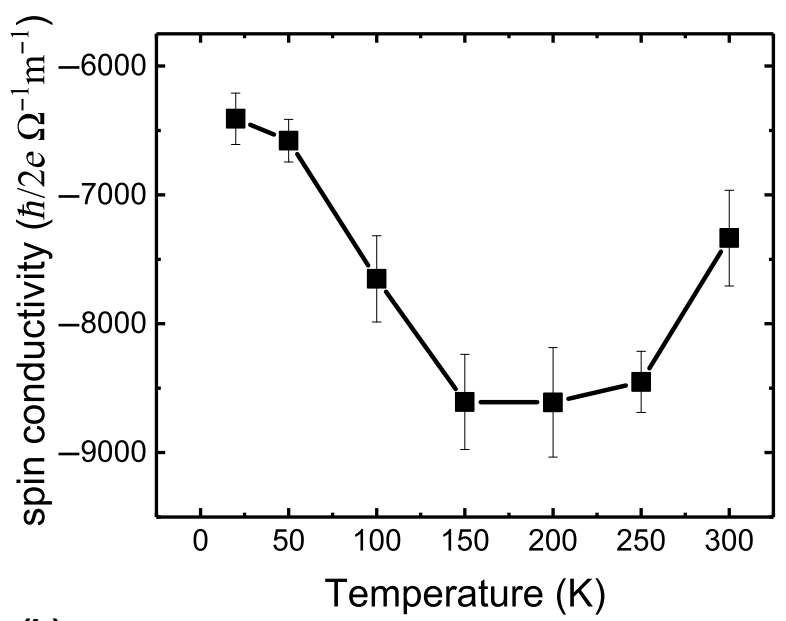

(b)

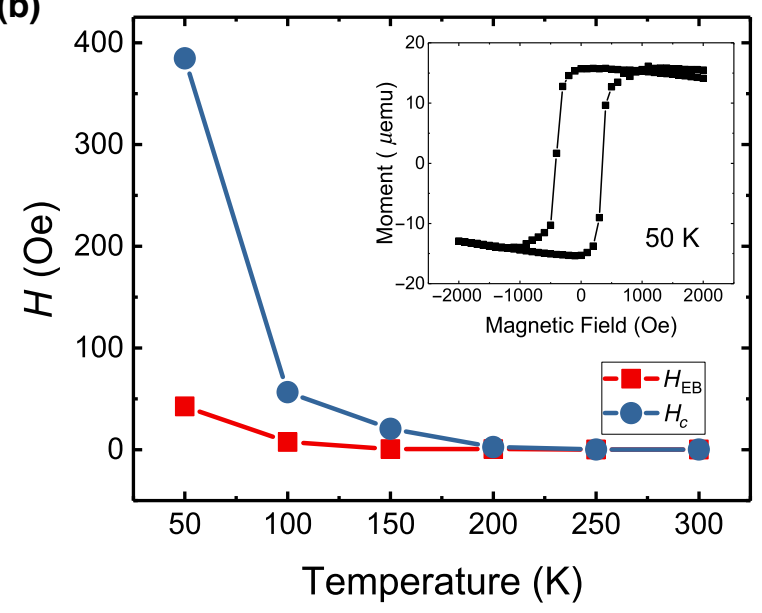

FIG. 3. (a) Spin conductivity measured at different temperatures for Ir-Mn 1.0 sample. (b) Exchange bias and coercivity measured at different temperatures for the Ir-Mn (1.0)/Ni-Fe (3.0)/Ru (5) (thickness in nanometers) sample. The inset shows a typical exchange-biased hysteresis loop.

$\left(T / T_{N}\right)^{2}$ below the Néel temperature $[14,45]$. In contrast, the magnon conductivity increases with temperature (more magnons become available), while the magnon relaxation length decreases due to the progressive loss of antiferromagnetic order. The interplay between these two effects results in a smooth, rather than abrupt, enhancement of the spin conductivity around the Néel temperature. Above the Néel temperature, the magnonic transport is mediated by paramagnons supported by thermal fluctuations. Therefore, the spin conductivity is still pronounced above the Néel temperature but decreases upon further increasing temperature, as observed in Fig. 3(a).

\section{Dependence of spin conductivity on AFM layer thickness}

The temperature-dependent spin conductivity measured from samples of different thicknesses of the Ir-Mn layer is shown in Fig. 4 (a). Remarkably, the absolute value of the negative spin conductivity is significantly larger in the Ir-Mn 1.0 and 1.5 samples than in the Ir-Mn 0 sample. Upon increasing the Ir-Mn thickness further, the absolute value of the negative spin conductivity reduces progressively and changes sign at a thickness between 3 and $4 \mathrm{~nm}$ Ir-Mn. The positive spin conductivity seems saturated at a thickness of 8 or $10 \mathrm{~nm}$. This sign change is expected, since Ta and Ir-Mn provide opposite spin Hall effects $[5,25]$ to the FM layer and their spin Hall effects have different temperature dependencies. We note that the spin conductivity is not close to zero at low temperatures, which is different from the pure magnonic spin current in AFM insulators [10,14]. As shown in Fig. 4(a), we still observe the negative peak of spin conductivity at $1.5 \mathrm{~nm}$. At low temperatures, we expect the electronic spin current to dominate, leading to a nonzero spin conductivity due to the small number of magnons created by spin fluctuation,
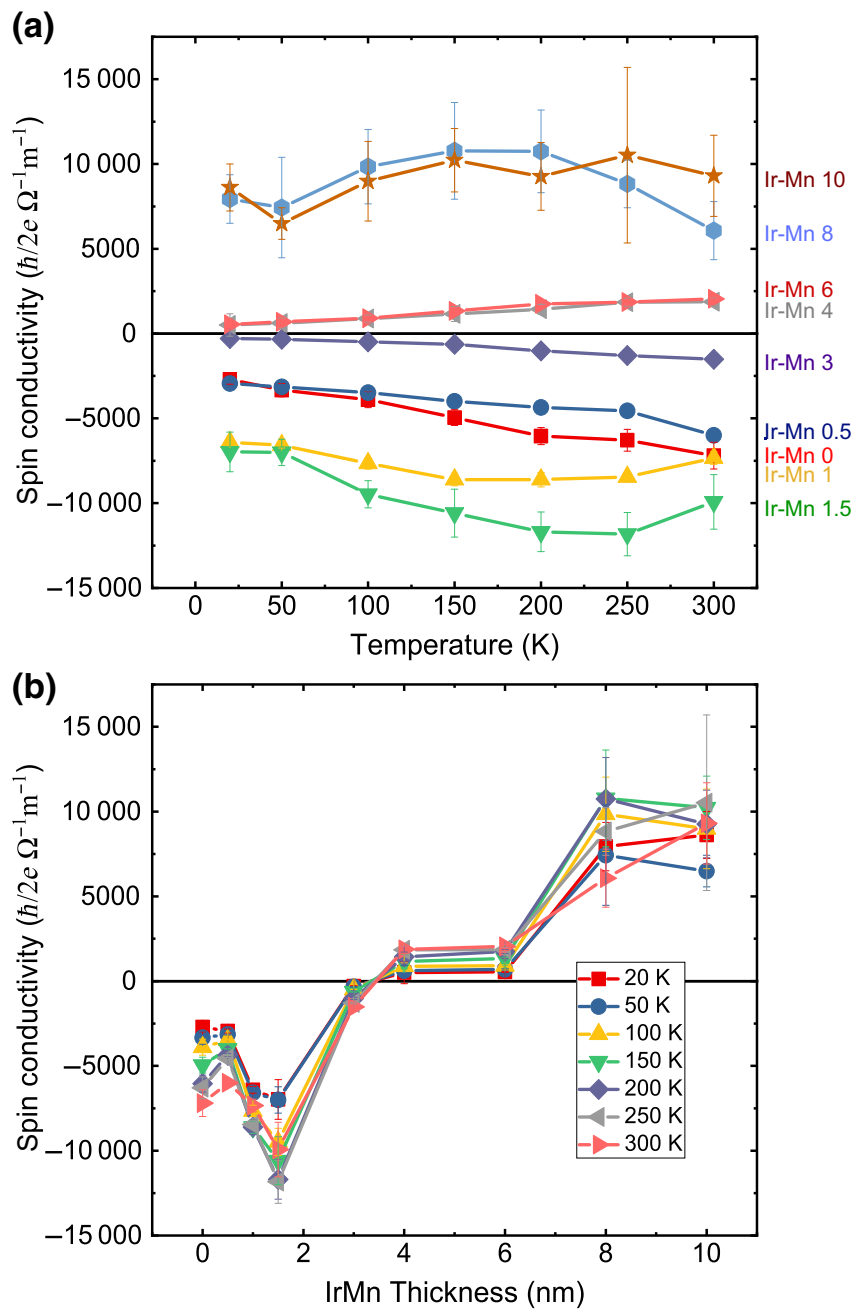

FIG. 4. (a) Spin conductivity as a function of temperature for samples with different Ir-Mn thicknesses. (b) Spin conductivity as a function of Ir-Mn thickness at various temperatures. 
which is in sharp contrast with previous reports on AFM insulators [14].

The systematic variation of spin Hall conductivity as a function of Ir-Mn thickness measured at various temperatures is presented in Fig. 4(b). We can distinguish three regions. From $t_{\mathrm{Ir}-\mathrm{Mn}}=0$ to $0.5 \mathrm{~nm}$, the spin conductivity is negative, and its absolute value slightly decreases upon increasing Ir-Mn thickness (except at $20 \mathrm{~K}$ ). The electronic spin current is scattered because of the imperfect interface, while magnons are unlikely to be present due to the lack of the AFM order. In the second region, the spin conductivity increases sharply with increasing Ir-Mn thickness from 0.5 to $1.5 \mathrm{~nm}$. In the third region, as the thickness of Ir-Mn increases further from $1.5 \mathrm{~nm}$, the absolute value of the negative spin conductivity quickly approaches a very small value at $t_{\mathrm{Ir}-\mathrm{Mn}}=3 \mathrm{~nm}$. Due to the strong electron spin relaxation in $\operatorname{Ir}-\mathrm{Mn}(<1 \mathrm{~nm})$, the contribution of the electronic spin current is likely to progressively disappear, while the magnonic contribution is expected to increase with the establishment of the AFM order. When the thickness of Ir-Mn exceeds $2 \mathrm{~nm}$, the spin conductivity decreases to zero and then changes sign. Because Ta and Ir-Mn have opposite spin Hall angles, the sign change indicates the dominating spin source is changed from the Ta layer to the Ir-Mn layer. This decrease in spin conductivity suggests that it becomes more and more difficult for the magnonic spin current, which carries the spin information from the Ta layer, to penetrate the Ir-Mn layer when the thickness of Ir-Mn increases. Hence, the reduction in spin conductivity suggests that the magnon relaxation length in Ir-Mn is rather short and may not exceed a few nanometers. Interestingly, in the intermediate region of $3 \mathrm{~nm}<t_{\mathrm{Ir}-\mathrm{Mn}}<7 \mathrm{~nm}$, the spin conductivity displays a plateau, which is in contrast with that reported in Ref. [9]. To better understand these features and extract the magnon transport parameters, we now turn our attention toward the theoretical model of the heterostructure.

\section{THEORETICAL MODELING}

The conversion between electron-mediated and magnonmediated spin currents at the interface between a metal and a FM [45,46] or an AFM [47-51] insulator has been previously studied theoretically. These works focused on magnetic insulators, where electronic spin current is quenched, and particularly addressed the electron-magnon conversion driven by a temperature gradient across the interface. Here, we are interested in current-driven spin transport across all-metallic systems while considering a homogeneous temperature.

To understand the experimental results, we consider the ideal model depicted in Fig. 5. A charge current $J_{c}$ flows homogeneously in the plane of the heterostructure along the $y$ axis (black arrow). In the heavy metal (HM, gray layer) and in the antiferromagnet (AFM, coral layer), it

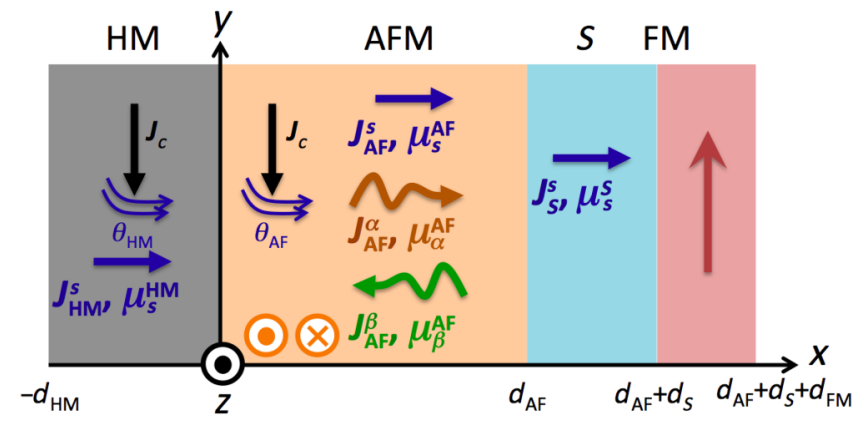

FIG. 5. Schematics of the model magnetic multilayer. The heterostructure is composed of four layers: a heavy metal (HM gray) providing SHE with efficiency $\theta_{\mathrm{HM}}$, an antiferromagnetic layer (AFM, coral) providing SHE with efficiency $\theta_{\mathrm{AF}}$ and with an order parameter along $z$ (out-of-plane orange arrows) enabling magnon propagation (brown and green wavy arrows), a light metal space ( $\mathrm{S}$, light blue), and finally a ferromagnet (FM, red) with magnetization along the $y$ axis (red arrow).

generates a nonequilibrium transverse spin current via the SHE, $J_{\mathrm{HM}, \mathrm{AF}}^{S}$. The spin Hall angle in each layer is $\theta_{\mathrm{HM}, \mathrm{AF}}$. In the AFM layer, we assume that the order parameter is fixed along the $z$ direction, as represented by the out-of-plane orange arrows. This configuration enables the excitation of nonequilibrium AFM magnons. Therefore, the incoming electronic spin current $J_{\mathrm{HM}}^{s}$ in the heavy metal is converted into an electronic spin current $J_{\mathrm{AF}}^{s}$ as well as a magnonic spin current $J_{\mathrm{AF}}^{\alpha, \beta}$, where $\alpha, \beta$ refer to the two magnon modes of the AFM. These three spin currents then convert again into an electronic spin current $J_{S}^{S}$ in the spacer layer ( $\mathrm{S}$, light blue layer), which then exerts a torque on the magnetization of the ferromagnet (FM, red layer). We emphasize that this situation does not accurately reflect the experimental situation since Ir-Mn is sputtered and is likely to be constituted of disordered AFM grains. Henceforth, only the grains with an AFM order parameter aligned along $z$ convey magnonic currents.

Let us now compute the spin current transmitted from the HM layer to the FM via the AFM layer. The boundary conditions and drift-diffusion model are derived in Sec. IV A following Refs. [45] and [49]. Materials' parameters are discussed in Sec. IV B and the model is analyzed and compared to the experimental data in Sec. IV C.

\section{A. Spin torque mediated by antiferromagnet}

\section{Boundary condition: spin conductance and spin convertance}

We consider a bipartite AFM with uniaxial anisotropy for simplicity (see Appendix A 1). As discussed in Sec. II, our Ir-Mn is likely to adopt the noncollinear $\gamma$ phase. Accounting for such a magnetic texture would simply 
make the overall system more complex, without qualitatively changing our conclusions. In the bipartite antiferromagnet, two eigenmodes $\alpha$ and $\beta$ couple to the electronic system at the interface between a normal metal and an AFM metal. There, the boundary conditions are (see Appendix A 2)

$$
\begin{aligned}
J_{\mathrm{HM}, S}^{s}= & J_{\mathrm{AF}}^{s}+J_{\mathrm{AF}}^{\alpha}+J_{\mathrm{AF}}^{\beta}, \\
J_{\text {tot }}^{s}= & G_{\alpha}\left(\Delta \mu_{s}-\mu_{\alpha}\right)+G_{\beta}\left(\Delta \mu_{s}+\mu_{\beta}\right) \\
& +G_{\text {int }} \Delta \mu_{s}, \\
J^{\alpha}= & G_{\alpha}\left(\Delta \mu_{s}-\mu_{\alpha}\right), \\
J^{\beta}= & G_{\beta}\left(\Delta \mu_{s}+\mu_{\beta}\right) .
\end{aligned}
$$

$G_{\text {int }}$ and $G_{\alpha, \beta}$ are the interfacial electronic spin conductance and electron-magnon spin convertance [45], respectively, $\Delta \mu_{s}=\mu_{s}^{-}-\mu_{s}^{+}$is the spin-dependent electronic chemical potential difference across the interface, and $\mu_{\alpha, \beta}$ are the magnon chemical potentials in the antiferromagnet. The electron-magnon spin convertance explicitly reads [45]

$$
G_{\alpha, \beta}=\frac{2 \pi \mu_{B} a^{7}}{\hbar} g_{F}^{2} \Lambda_{\alpha, \beta}^{2} \int \omega\left(-\partial_{\omega} n_{0}\right) g_{m}^{\alpha, \beta}(\omega) d \omega .
$$

In the above expression, $g_{F}$ and $g_{m}^{\alpha, \beta}$ are the electron and magnon densities of states, $n_{0}$ is the Bose-Einstein distribution, $a$ is the lattice parameter, $\omega$ is the magnon energy, and $\Lambda_{\alpha, \beta}^{2}$ scales with the interfacial $s$ - $d$ exchange interaction $J_{s-d}$ (see Appendix A 2 b). Equation (3) states the continuity of spin current at interfaces (no interfacial spin-flip), while Eqs. (4)-(6) are the generalized Kirchhoff's laws for the total and magnonic spin currents. The boundary conditions Eqs. (3)-(6) apply to both the left and right interfaces of the AFM metal.

\section{Magnon and electron spin diffusion equations}

We assume the magnon transport in the AFM layer is diffusive [45], such that the magnon-mediated spin current and magnon accumulations read

$$
\begin{aligned}
J^{\alpha, \beta} & =\mp 2 \mu_{B} \sigma_{\alpha, \beta} \partial_{x} \mu_{\alpha, \beta}, \\
\frac{\mu_{\alpha, \beta}}{\left(l_{\alpha, \beta}\right)^{2}} & =\partial_{x}^{2} \mu_{\alpha, \beta},
\end{aligned}
$$

with the magnon conductivity $\sigma_{\alpha, \beta}=\left(\tau_{\text {th }} / 3\right) \int v^{2} g_{m}^{\alpha, \beta}(\omega)$ $\left(-\partial_{\omega} n_{0}\right) d \omega$ and the magnon diffusion length $\left(l_{\alpha, \beta}\right)^{2}$ $=\left[\tau_{\mathrm{th}} \sigma_{\alpha, \beta} / \int g_{m}^{\alpha, \beta}(\omega)\left(-\partial_{\omega} n_{0}\right) d \omega\right]$. Here $v$ is the magnon velocity and $\tau_{\text {th }}$ is the magnon thermalization time. The explicit forms of the electron-magnon spin convertance, conductivity, and diffusion length are given in Appendix A 3. Assuming the dispersion, velocity, and density of states of a uniaxial body centered cubic antiferromagnetic lattice (see Appendix A 1), as well as a weak anisotropy energy $\omega_{K}$ and a large Néel temperature $\left(\int_{\omega_{K}}^{k_{B} T_{N}} d \omega \rightarrow \int_{0}^{+\in f y} d \omega\right)$, the scaling of these three quantities is

$$
\begin{aligned}
G_{\alpha, \beta} & \sim\left(\frac{J_{s d}}{\mu_{B} \mu_{0} H_{E}}\right)^{2} \frac{g_{F}^{2}}{\hbar \mathcal{A}} k_{B}^{2} T^{2}, \\
\sigma_{\alpha, \beta} & \sim \frac{\tau_{\text {th }} / \hbar}{\mu_{B} \mu_{0} H_{E}} \frac{k_{B}^{2} T^{2}}{\hbar a} \\
l_{\alpha, \beta} & \sim a \frac{\tau_{\text {th }}}{\hbar} \mu_{B} \mu_{0} H_{E} .
\end{aligned}
$$

Here, $H_{E}$ is the antiferromagnetic exchange field and $\mathcal{A}$ is the area of the interfacial unit cell. As shown in Sec. IV C, the physical quantity that controls the injection of magnons in the antiferromagnetic layer is $\left(G_{\alpha, \beta} l_{\alpha, \beta} / \sigma_{\alpha, \beta}\right) \sim g_{F}^{2} J_{s-d}^{2}$, which is mostly temperature independent.

Let us now define the drift-diffusion equation of the electronic and magnonic spins in the various layers. In the HM layer, the electronic spin current polarized along $z$ and flowing along $x$ reads $e J_{\mathrm{HM}}^{s}=-\sigma_{\mathrm{HM}} \partial_{x} \mu_{s}^{\mathrm{HM}}+\sigma_{\mathrm{HM}} \theta_{\mathrm{HM}} E$, while the spin accumulation fulfills $\partial_{x}^{2} \mu_{s}^{\mathrm{HM}}=\mu_{s}^{\mathrm{HM}} / \lambda_{\mathrm{HM}}^{2}$. Here, $\sigma_{\mathrm{HM}}$ and $\lambda_{\mathrm{HM}}$ are the electronic conductivity and spin diffusion length in the heavy metal. The same law applies in the AFM and in the spacer, although the latter does not display SHE $\left(\theta_{\mathrm{AF}} \neq 0, \theta_{S}=0\right)$. In addition, the magnon spin current fulfills the usual drift-diffusion equations, Eqs. (8) and (9), and we assume that the $\alpha$ and $\beta$ magnons display the same properties, although they carry opposite $\operatorname{spin}\left(\sigma_{\alpha}=\sigma_{\beta}, l_{\alpha}=l_{\beta}, G_{\alpha}=G_{\beta}\right)$. Breaking the degeneracy of these two modes severely enhances the complexity of the analytical treatment.

\section{Determination of the spin Hall torque}

Having established the transport equations and boundary conditions of our system, we now proceed with computing the spin Hall torque in the heterostructure. At the left outer boundary, $x=-d_{\mathrm{HM}}$, we impose that no electronic spin current flows out of the system, $J_{\mathrm{HM}}^{s}=0$. At the HM/AFM interface, $x=0$, we apply the conditions Eqs. (3)-(6). Since both magnon modes are degenerate, we obtain $\mu_{\beta}=-\mu_{\alpha}$ and

$$
\begin{aligned}
\left.J_{\text {tot }}^{s}\right|_{x=0}= & 2 G_{\alpha}^{L}\left(\mu_{s}^{\mathrm{HM}}-\mu_{s}^{\mathrm{AF}}-\mu_{\alpha}^{\mathrm{AF}}\right) \\
& +G_{\text {int }}^{L}\left(\mu_{s}^{\mathrm{HM}}-\mu_{s}^{\mathrm{AF}}\right), \\
\left.J^{\alpha}\right|_{x=0}= & G_{\alpha}^{L}\left(\mu_{s}^{\mathrm{HM}}-\mu_{s}^{\mathrm{AF}}-\mu_{\alpha}^{\mathrm{AF}}\right),
\end{aligned}
$$

where the total spin current is continuous and reads

$$
\left.J_{\text {tot }}^{s}\right|_{x=0}=\left.J_{\mathrm{HM}}^{S}\right|_{x=0^{-}}=\left.J_{\mathrm{AF}}^{s}\right|_{x=0^{+}}+\left.2 J_{\mathrm{AF}}^{\alpha}\right|_{x=0^{+}} .
$$

At the AFM/S boundary, $x=d_{\mathrm{AF}}$, we apply the same boundary conditions. Notice that the spacer $(\mathrm{Cu}$ in the 
experiment) has a very large (infinite) spin diffusion length, which means that the spin current at the AFM/S interface is unaltered and fully absorbed by the FM layer. In other words, the electronic spin accumulation, transverse to the $\mathrm{FM}$ order parameter, vanishes at the $\mathrm{AFM} / \mathrm{S}$ interface, $\mu_{s}^{S}=0$. This yields the following boundary conditions

$$
\begin{aligned}
\left.J_{\text {tot }}^{s}\right|_{x=d_{\mathrm{AF}}} & =2 G_{\alpha}^{R}\left(\mu_{s}^{\mathrm{AF}}+\mu_{\alpha}^{\mathrm{AF}}\right)+G_{\mathrm{int}}^{R} \mu_{s}^{\mathrm{AF}}, \\
\left.J^{\alpha}\right|_{x=d_{\mathrm{AF}}} & =G_{\alpha}^{R}\left(\mu_{s}^{\mathrm{AF}}+\mu_{\alpha}^{\mathrm{AF}}\right) .
\end{aligned}
$$

In the simulations, and to keep the system as simple as possible, we consider symmetric interfaces, $G_{\alpha, \text { int }}^{R}=G_{\alpha, \text { int }}^{L}$. The spin Hall torque exerted on the FM layer is simply given by the spin current at the AFM/S interface

$$
T=2 G_{\alpha}^{R}\left(\mu_{s}^{\mathrm{AF}}+\mu_{\alpha}^{\mathrm{AF}}\right)+G_{\text {int }}^{R} \mu_{s}^{\mathrm{AF}} .
$$

\section{B. Materials' parameters}

The materials' parameters of metals for electronic transport are well known [5,25]. For the simulations, we use typical materials' parameters for $\mathrm{Ta}$ $\left(\sigma_{\mathrm{Ta}}=4.3 \times 10^{5} \Omega^{-1} \mathrm{~m}^{-1}, \lambda_{\mathrm{Ta}}=5 \mathrm{~nm}, \theta_{\mathrm{Ta}} \approx-0.12\right)$ and Ir-Mn $\quad\left(\sigma_{\text {Ir-Mn }}=8.6 \times 10^{5} \Omega^{-1} \quad \mathrm{~m}^{-1}, \lambda_{\mathrm{Ir}-\mathrm{Mn}}=1.4 \mathrm{~nm}\right.$, $\left.\theta_{\mathrm{Ir}-\mathrm{Mn}} \approx 0.01\right)$ and assume $G_{\mathrm{Ta} / \mathrm{Ir}-\mathrm{Mn}}=10^{15} \Omega^{-1} \mathrm{~m}^{-2}$ $[23,52]$ (see Table I). However, the parameters for magnon transport have not been systematically tabulated yet. Szunyogh et al. [53] have computed the magnetic interactions of crystalline Ir-Mn $\mathrm{Mn}_{3}$ in the $L 1_{2}$ phase and obtained an AFM exchange interaction of $\mu_{B} \mu_{0} H_{E}=40 \mathrm{meV}$ and a uniaxial anisotropy energy $\mu_{B} \mu_{0} H_{K} \approx 10 \mathrm{meV}$. The magnon thermalization time $\tau_{\text {th }}$ is, by definition, inversely proportional to the magnetic damping. For instance, the Gilbert damping of YIG is approximately $10^{-4}$ while the magnon relaxation time is estimated to be $\tau_{\mathrm{th}}^{\mathrm{YIG}} \sim$
$10^{-6} \mathrm{~s}$ [45]. Hence, in Ir-Mn, a heavy transition metal, the magnetic damping is expected to be around 0.1 , and therefore, we assume a magnon relaxation time of $\tau_{\mathrm{th}}^{\mathrm{Ir}-\mathrm{Mn}} \sim$ $10^{-9} \mathrm{~s}$. The exchange $J_{s d}$ between itinerant and localized spins is more difficult to extract from first-principle calculations. One approach is to consider the spin polarization at the Fermi level per sublattice. Investigating $\mathrm{Fe}-\mathrm{Mn}, \mathrm{Xu}$ et al. [54] computed a spin-polarization of about $10 \%$ on $\mathrm{Mn}$ sites. Assuming a polarization $P=$ $\left(J_{s d} / \epsilon_{F}\right)=10 \%\left(\epsilon_{F}\right.$ is the Fermi energy $)$, a free electron density of states $g_{F}=\left(a^{3} / 2 \pi^{2}\right)(2 m / \hbar)^{3 / 2} \sqrt{\epsilon_{F}} \approx 1 \mathrm{eV}^{-1}$ and the lattice parameter of crystalline Ir- $\mathrm{Mn}_{3} a_{\mathrm{IrMn}}=$ $3.78 \AA$, we obtain $J_{s-d}=0.6 \mathrm{eV} \gg \mu_{B} \mu_{0} H_{E}$. Assuming room temperature $\left(k_{B} T=25 \mathrm{meV}\right)$, one finds $G_{\alpha} \approx 4.2 \times$ $10^{14} \Omega^{-1} \mathrm{~m}^{-2}, \sigma_{\alpha} \approx 1.7 \times 10^{9} \Omega^{-1} \mathrm{~m}^{-1}$, and $l_{\alpha} \approx 12 \mu \mathrm{m}$ (notice that Zhang and Zhang [45] estimated $l_{\alpha} \approx 1 \mathrm{~mm}$ in YIG). These numbers are computed in the ideal case of a well-ordered AFM with very large exchange interaction, while the one considered in the experiment is obtained by sputtering and potentially exhibits a high degree of (magnetic and crystalline) disorder.

Finally, we emphasize that the degree of magnetic ordering depends on the thickness of the AFM layer [25]. To account for this thickness dependence phenomenologically, and in the absence of further information, we choose to keep the magnon diffusion length and bulk conductivity, $\sigma_{\alpha}$ and $\lambda_{\alpha}$, constant while the spin convertance $G_{\alpha}$ is assumed to adopt the form

$$
G_{\alpha}\left(d_{\mathrm{AF}}\right)=G_{\alpha}^{0}\left[1+\tanh \left(d_{\mathrm{AF}}-d_{\mathrm{AF}}^{o}\right) / l_{o}\right] .
$$

Here, $d_{\mathrm{AF}}^{o}$ is the thickness at which the magnetic order sets up and $l_{o}$ is the distance over which the system passes from a disordered paramagnet to an ordered AFM. We emphasize that this dependence is phenomenological and only

TABLE I. Materials parameters for the heavy metal and antiferromagnet in the two experimental cases of interest.

\begin{tabular}{lcc}
\hline \hline Electronic parameters & $\mathrm{Ta} / \mathrm{Ir}-\mathrm{Mn} / \mathrm{Cu} / \mathrm{Ni}-\mathrm{Fe}$ (This work) & W/Fe-Mn/Ni-Fe [9] \\
\hline$\sigma_{\mathrm{HM}}\left(\Omega^{-1} \mathrm{~m}^{-1}\right)$ & $4.3 \times 10^{5}$ & $5 \times 10^{5}$ \\
$\lambda_{\mathrm{HM}}(\mathrm{nm})$ & 2 & 2 \\
$\theta_{\mathrm{HM}}$ & -0.12 & -0.3 \\
$d_{\mathrm{HM}}(\mathrm{nm})$ & 5 & 4 \\
$\sigma_{\mathrm{AF}}\left(\Omega^{-1} \mathrm{~m}^{-1}\right)$ & $8.3 \times 10^{5}$ & $6 \times 10^{5}$ \\
$\lambda_{\mathrm{AF}}(\mathrm{nm})$ & 1.6 & 1.8 \\
$\theta_{\mathrm{AF}}$ & 0.01 & 0.01 \\
Adjustable parameters & $5 \times 10^{6}$ & $7.7 \times 10^{6}$ \\
$\sigma_{\alpha}\left(\Omega^{-1} \mathrm{~m}^{-1}\right)$ & 6 & 6 \\
$\lambda_{\alpha}(\mathrm{nm})$ & $10^{15}$ & $10^{14}$ \\
$G_{\mathrm{int}}\left(\Omega^{-1} \mathrm{~m}^{-2}\right)$ & $3 \times 10^{16}$ & $1.6 \times 10^{15}$ \\
$G_{\alpha}^{0}\left(\Omega^{-1} \mathrm{~m}^{-2}\right)$ & 6 & 7 \\
$d_{\mathrm{AF}}^{0}\left(\Omega^{-1} \mathrm{~m}^{-2}\right)$ & 1 & 1.6 \\
$\lambda_{c}(\mathrm{~nm})$ & \\
\hline \hline
\end{tabular}

The electronic parameters are fixed and the adjustable parameters are tuned to obtain the best fit possible. 
aims at representing the experimental fact that antiferromagnetism sets up above a certain critical thickness. We have tested various scenarios, such as making both $G_{\alpha}$ and $\sigma_{\alpha}$ thickness dependent, without qualitatively changing the results discussed in the next section.

\section{Spin hall torque simulations}

\section{Thickness dependence}

Before attempting to fit the experimental data, let us first explore the thickness dependence of the spin Hall torque in various situations. Figure 6 shows the thickness dependence for three values of the magnon conductivity and setting $\lambda_{\alpha}=6 \mathrm{~nm}$. In Fig. 6(a), the magnon conductivity is small, $\sigma_{\alpha}=10^{6} \Omega^{-1} \mathrm{~m}^{-1}$, such that the thickness dependence is weakly modified upon turning on the spin convertance. It displays the approximately $e^{-d_{\mathrm{AF}} / \lambda_{\mathrm{AF}}}$ law expected in a system entirely dominated by electronic transport and given by the black curve, $G_{\alpha}^{0}=10^{14} \ll$ $G_{\text {int }}=10^{15}$. In this limit (solid black line), the torque for $d_{\mathrm{AF}}=0$ and $d_{\mathrm{AF}}=+\infty$ can be easily computed

$$
T\left(d_{\mathrm{AF}}=0\right)=-\frac{\sigma_{\mathrm{HM}} \theta_{\mathrm{HM}} E\left[1-\cosh ^{-1}\left(d_{\mathrm{HM}} / \lambda_{\mathrm{HM}}\right)\right]}{1+\left(\sigma_{\mathrm{HM}} / \lambda_{\mathrm{HM}} G_{\mathrm{int}}\right) \tanh \left(d_{\mathrm{HM}} / \lambda_{\mathrm{HM}}\right)},
$$

$T\left(d_{\mathrm{AF}}=+\infty\right)=\frac{\sigma_{\mathrm{AF}} \theta_{\mathrm{AF}} E}{1+\left(\sigma_{\mathrm{AF}} / \lambda_{\mathrm{AF}} G_{\mathrm{int}}\right)}$.

In the absence of an AFM layer, the torque is solely given by the SHE in the HM layer [Eq. (20)], while for an infinitely thick AFM layer, it is solely given by the SHE in the AFM [Eq. (21)].

In Fig. 6(b), the magnon conductivity is larger, $\sigma_{\alpha}=$ $10^{7} \Omega^{-1} \mathrm{~m}^{-1}$, and one clearly sees a negative peak emerging progressively upon increasing the spin convertance. This peak characterizes the onset of magnon transport. While the electronic spin transport is quenched after a few nanometers $\left(\lambda_{\mathrm{AF}} \approx 1.4 \mathrm{~nm}\right)$, the magnetic order progressively builds up and is established around $5 \mathrm{~nm}$ in the present model, inducing a temporary recovery of the torque at this thickness. Upon further increase of the AFM thickness, the magnon diffusion takes over. At large thicknesses, it prevents the transmission of magnonic spin current from the HM layer to the FM layer. This effect results in a reversal of the torque, which is then dominated by the SHE of the AFM layer at large thicknesses.

In Fig. 6(c), yet another scenario is offered. In this plot, an even larger magnon conductivity is taken, $\sigma_{\alpha}=$ $10^{8} \Omega^{-1} \mathrm{~m}^{-1}$, and the spin torque goes continuously from negative to positive values without passing by a local negative maximum. While the weak spin convertance case, $G_{\alpha}^{0}=10^{14}$ (red curve), gives a similar result as Figs. 6(a) and 6(b), upon increasing the spin convertance, the torque exhibits an inflection point at the thickness around which the magnetic order sets up (approximately $5 \mathrm{~nm}$ ). It is also

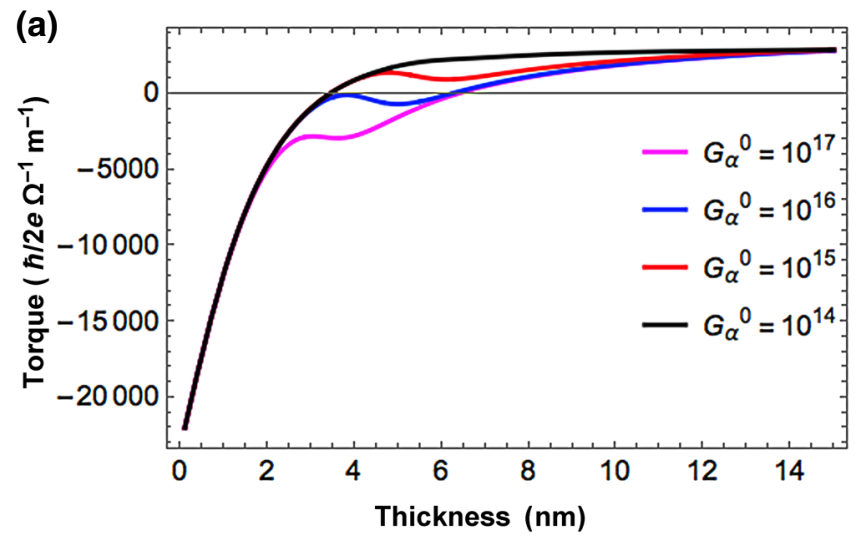

(b)

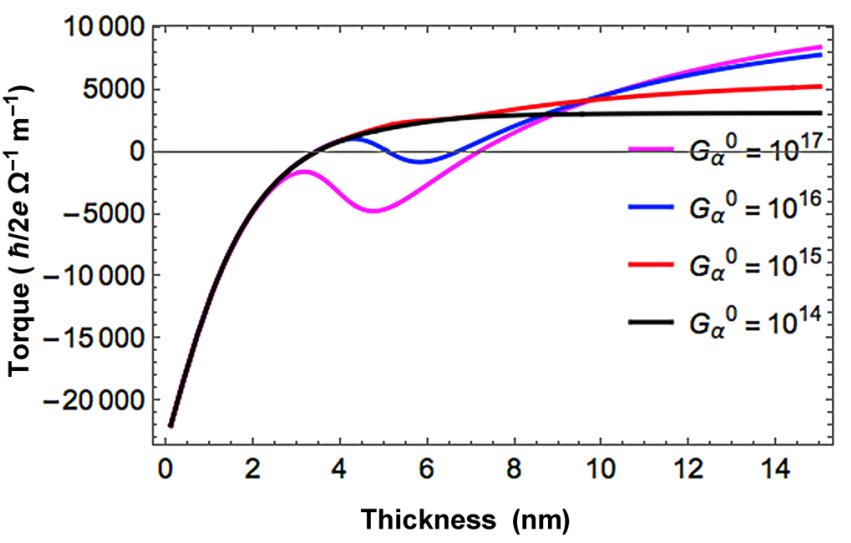

(c)

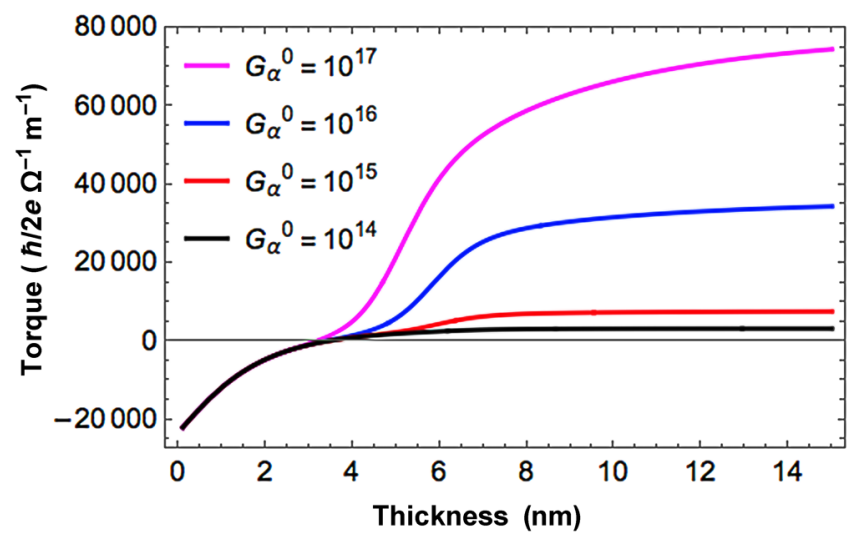

FIG. 6. Spin torque as a function of the antiferromagnetic thickness for $\lambda_{\alpha}=6 \mathrm{~nm}$ and (a) $\sigma_{\alpha}=10^{6} \Omega^{-1} \mathrm{~m}^{-1}$, (b) $\sigma_{\alpha}=$ $10^{7} \Omega^{-1} \mathrm{~m}^{-1}$, and (c) $\sigma_{\alpha}=10^{8} \Omega^{-1} \mathrm{~m}^{-1}$. The curves are calculated for various spin convertances $G_{\alpha}^{0}$. The units are given in $\Omega^{-1} \mathrm{~m}^{-2}$.

remarkable that for large thicknesses, the torque reaches a much larger positive maximum than in Figs. 6(c) and 6(b). This positive value scales with the spin convertance $G_{\alpha}^{0}$. This behavior indicates a different situation compared to Fig. 6(b). At large thicknesses, when the electronic and magnonic spin currents coming from the HM layer are quenched, the spin Hall from the AFM is the only source of 
(a)

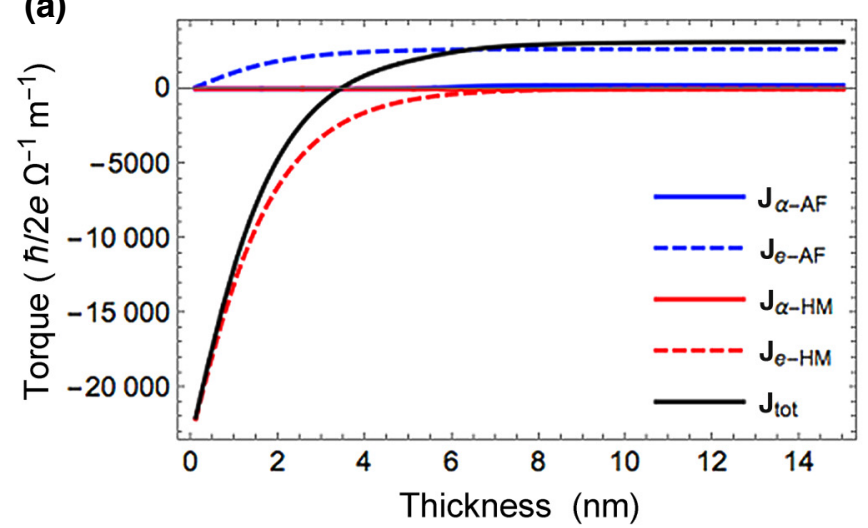

(b)

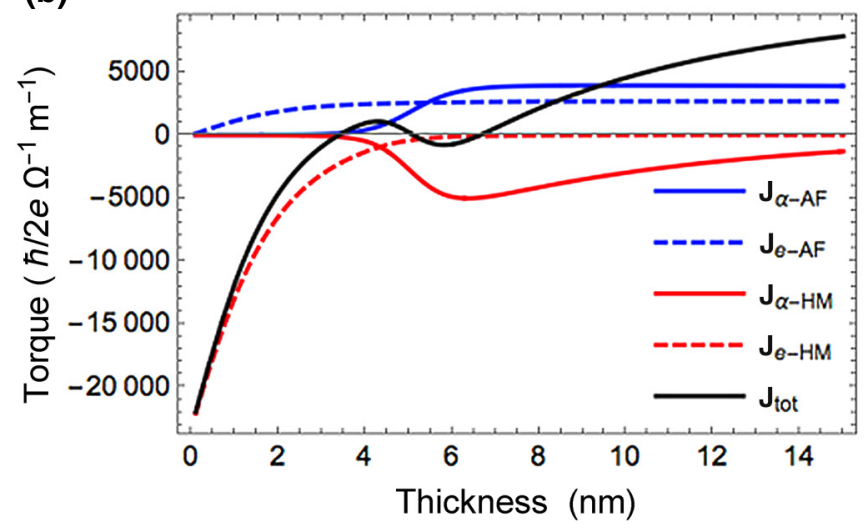

(c)

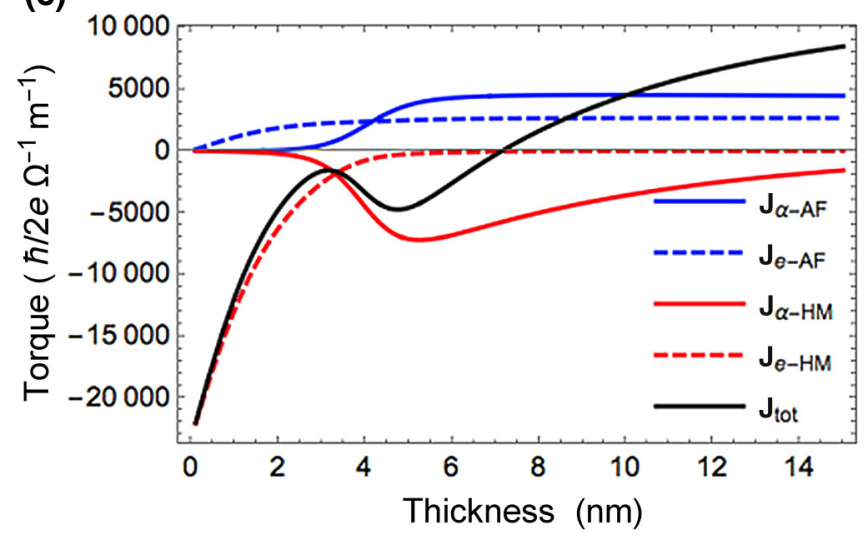

(d)

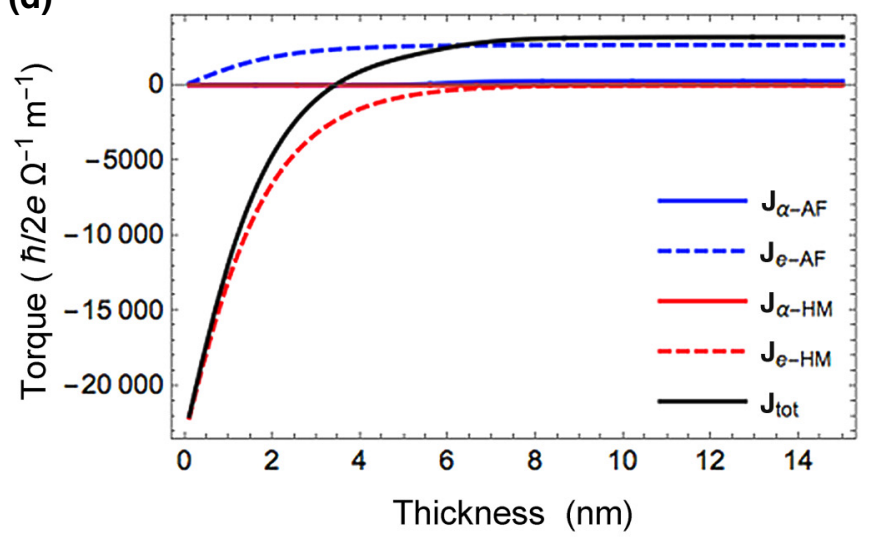

(e)

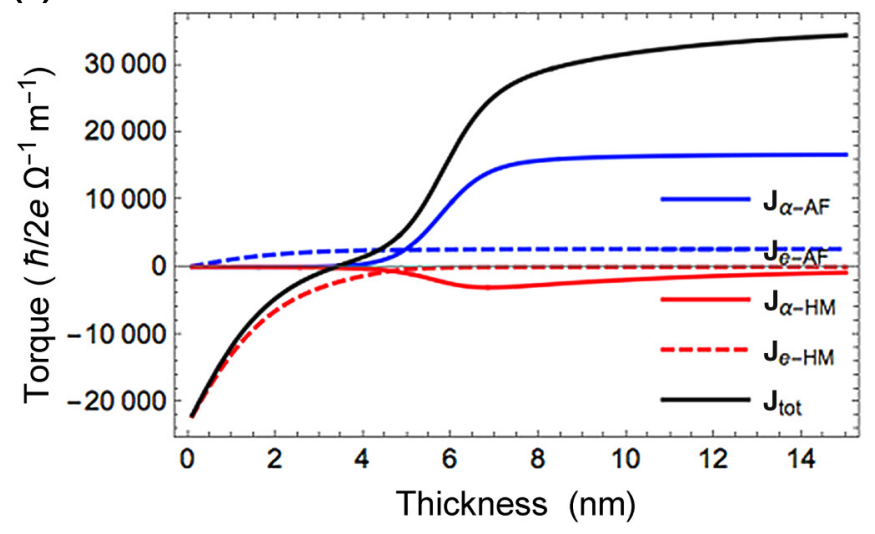

(f)

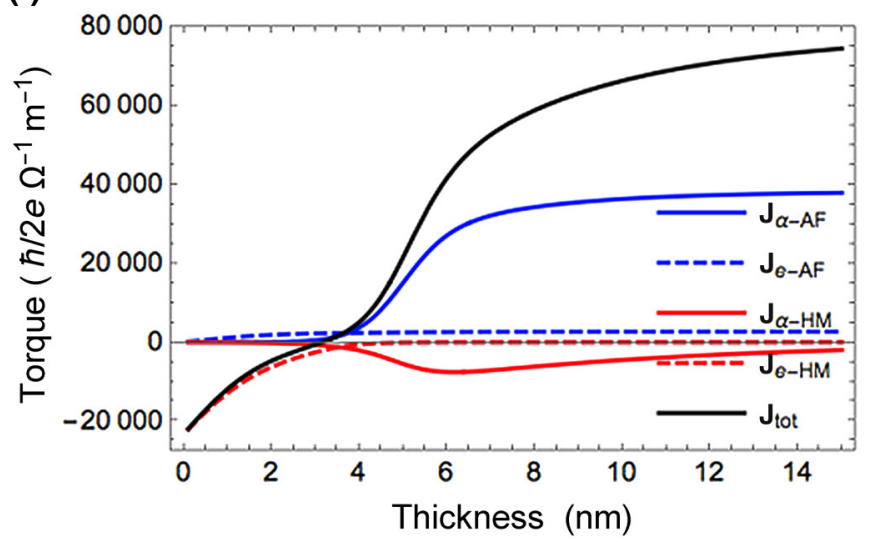

FIG. 7. Anatomy of the torque as a function of the AFM thickness for $\lambda_{\alpha}=6 \mathrm{~nm}, \sigma_{\alpha}=10^{7} \Omega^{-1} \mathrm{~m}^{-1}$, (a) $G_{\alpha}^{0}=10^{14} \Omega^{-1} \mathrm{~m}^{-2}$, (b) $G_{\alpha}^{0}=10^{16} \Omega^{-1} \mathrm{~m}^{-2}$, and (c) $G_{\alpha}^{0}=10^{17} \Omega^{-1} \mathrm{~m}^{-2}$, and $\sigma_{\alpha}=10^{8} \Omega^{-1} \mathrm{~m}^{-1}$, (d) $G_{\alpha}^{0}=10^{14} \Omega^{-1} \mathrm{~m}^{-2}$, (e) $G_{\alpha}^{0}=10^{16} \Omega^{-1} \mathrm{~m}^{-2}$, and (f) $G_{\alpha}^{0}=10^{17} \Omega^{-1} \mathrm{~m}^{-2}$. The black curve represents the total spin current (which equals the spin torque) at the AFM/S interface, the solid (dashed) blue lines represent the magnon (electronic) spin currents due to the SHE of the AFM layer, while the solid (dashed) red lines represent the magnonic (electronic) spin currents due to the SHE of the HM layer.

spin torque. Because $\sigma_{\alpha} \gg \sigma_{\mathrm{AF}}$, it is more efficient for the SHE to be transmitted via magnons. Therefore, the torque increases with the electron-magnon spin convertance at large thicknesses and can be much larger than when it is solely due to electronic transport. We discuss this effect in detail in the next subsection.

\section{Competition between electronic and magnonic spin currents}

To better understand the origin of the features identified above (local negative maximum and inflection point), we now analyze the four spin current contributions to the total torque: electronic and magnonic spin currents due to SHE 
in the HM layer, and electronic and magnonic spin currents due to SHE in the AFM layer. Figure 7 shows the results for different situations, (a,d) $G_{\alpha}^{0}=10^{14} \Omega^{-1} \mathrm{~m}^{-2}$, (b,e) $G_{\alpha}^{0}=10^{16} \Omega^{-1} \mathrm{~m}^{-2}$, and (c,f) $G_{\alpha}^{0}=10^{17} \Omega^{-1} \mathrm{~m}^{-2}$, taking (a-c) $\sigma_{\alpha}=10^{7} \Omega^{-1} \mathrm{~m}^{-1}$ and (d-f) $\sigma_{\alpha}=10^{8} \Omega^{-1} \mathrm{~m}^{-1}$. We compute the (dashed lines) electronic spin current and (solid lines) magnonic spin current at the AFM/S interface due to the SHE of the AFM layer (blue lines) or to the SHE of the HM layer (red lines).

In all cases considered, the thickness dependence of the electronic spin currents (dashed lines) displays the usual approximately $e^{-d_{\mathrm{AF}} / \lambda_{\mathrm{AF}}}$ law. In contrast, the thickness dependence of the contribution of the magnon-mediated spin Hall currents (solid lines) shows a complex variation arising from the combination of magnetic order setting up approximately $\left[1+\tanh \left(d_{\mathrm{AF}}-d_{\mathrm{AF}}^{0}\right) / \lambda_{c}\right]$ and magnon diffusionapproximately $e^{-d_{\mathrm{AF}} / \lambda_{\alpha}}$. When the magnon conductivity is comparable to the electronic conductivity, Figs. 7(a)-7(c), increasing the spin convertance results in an increase in magnon-mediated spin Hall current originating from the HM. The magnon-mediated spin Hall current originating from the AFM is also present, but is weakly sensitive to the increase in magnon conductivity. When increasing the magnon conductivity, the magnonmediated SHE from the AFM dominates, as displayed in Figs. 7(d)-7(f).

Figures 8(a) and 8(b) illustrate the two processes that occur when the magnon conductivity exceeds the electronic conductivity. At the interface between the HM layer and the AFM layer, Fig. 8(a), the SHE of the HM injects an electronic spin current into the AFM. When the electronmagnon spin convertance dominates over the electronic spin conductance, the magnonic spin current dominates over the electronic spin current. Solving the drift-diffusion model for such an interface in the limit $G_{\alpha},\left(2 \sigma_{\alpha} / \lambda_{\alpha}\right) \gg$ $G_{\text {int }}$, the magnon-mediated and electron-mediated spin
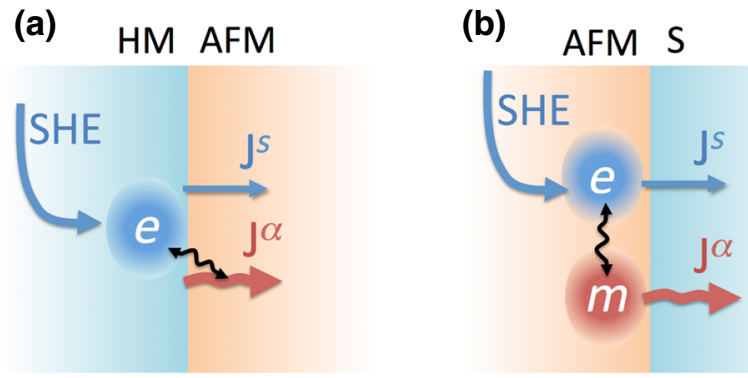

FIG. 8. Schematic of the electron-magnon conversion at (a) $\mathrm{HM} / \mathrm{AFM}$ and (b) AFM/S interfaces. The curved arrow represents the SHE that generates an electronic spin current and the straight blue (red) arrow represents the electronic (magnonic) spin current contributions. The shaded blue (red) puddles represent electronic (magnonic) spin accumulation at the interface and the wavy black arrow represents the electron-magnon interaction.

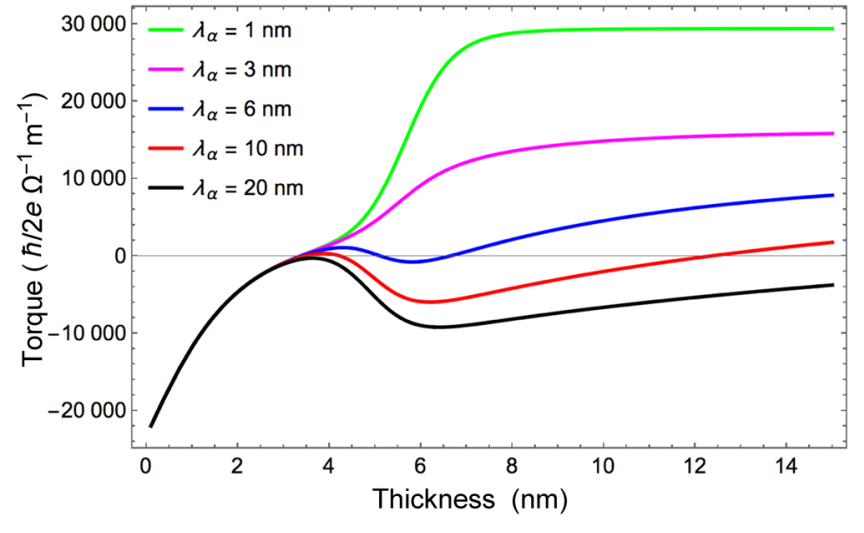

FIG. 9. Spin torque as a function of the antiferromagnetic thickness for different magnon diffusion lengths. Here, $\sigma_{\alpha}=$ $10^{7} \Omega^{-1} \mathrm{~m}^{-1}$ and $G_{\alpha}^{0}=10^{16} \Omega^{-1} \mathrm{~m}^{-2}$.

currents injected in the AFM read

$$
\begin{gathered}
J_{\mathrm{AF}}^{s}=\frac{G_{\mathrm{int}}}{2 G_{\alpha}} \frac{\sigma_{L} \lambda_{\mathrm{AF}}}{\sigma_{\mathrm{AF}} \lambda_{L}}\left(1+\frac{\lambda_{\alpha} G_{\alpha}}{\sigma_{\alpha}}\right) \sigma_{L} \theta_{L} E, \\
J_{\mathrm{AF}}^{\alpha}=\frac{\lambda_{\alpha} G_{\alpha}}{2 \sigma_{\alpha}} \sigma_{L} \theta_{L} E
\end{gathered}
$$

and one clearly sees that $J_{\mathrm{AF}}^{\alpha} \gg J_{\mathrm{AF}}^{s}$ in this limit, as expected intuitively. Meanwhile, at the interface between the AFM layer and the spacer, Fig. 8(b), the SHE in the AFM layer accumulates electronic spin density at the interface. Since the electron-magnon spin convertance exceeds the electronic spin conductance, this electronic spin density is first converted into a magnonic spin current, and then transmitted through the interface into an electronic spin current. The magnonic and electronic contributions to the total injected spin current (and therefore, to the overall torque exerted on the ferromagnet) read

$$
\begin{aligned}
J_{\mathrm{AF}}^{s} & =\frac{G_{\text {int }}}{2 G_{\alpha}} \frac{\sigma_{L} \lambda_{\mathrm{AF}}}{\sigma_{\mathrm{AF}} \lambda_{L}}\left(1+\frac{\lambda_{\alpha} G_{\alpha}}{\sigma_{\alpha}}\right) \sigma_{\mathrm{AF}} \theta_{\mathrm{AF}} E, \\
J_{\mathrm{AF}}^{\alpha} & =\frac{\sigma_{L} \lambda_{\mathrm{AF}}}{\sigma_{\mathrm{AF}} \lambda_{L}} \frac{\lambda_{\alpha} G_{\alpha}}{2 \sigma_{\alpha}} \sigma_{\mathrm{AF}} \theta_{\mathrm{AF}} E,
\end{aligned}
$$

and once again $J_{\mathrm{AF}}^{\alpha} \gg J_{\mathrm{AF}}^{s}$. Most importantly, one sees that in this limit, the spin torque is directly proportional to the spin convertance, $T=J_{\mathrm{AF}}^{s}+2 J_{\mathrm{AF}}^{\alpha} \approx 2 J_{\mathrm{AF}}^{\alpha} \sim G_{\alpha}$, which explains the increase observed in Fig. 6(c).

Equation (25) has to be compared with Eq. (21), which states that the electronic spin current only gives a torque $T=\eta \sigma_{\mathrm{AF}} \theta_{\mathrm{AF}} E$ where $\eta=\left[1+\left(\sigma_{\mathrm{AF}} / \lambda_{\mathrm{AF}} G_{\text {int }}\right)\right]^{-1}$ is sometimes called spin transparency. The magnon-mediated spin Hall torque has, therefore, the potential to overcome the obstacle of the interfacial electronic spin transparency.

To complete the present discussion, Fig. 9 displays the thickness dependence of the torque for various magnon 
(a)

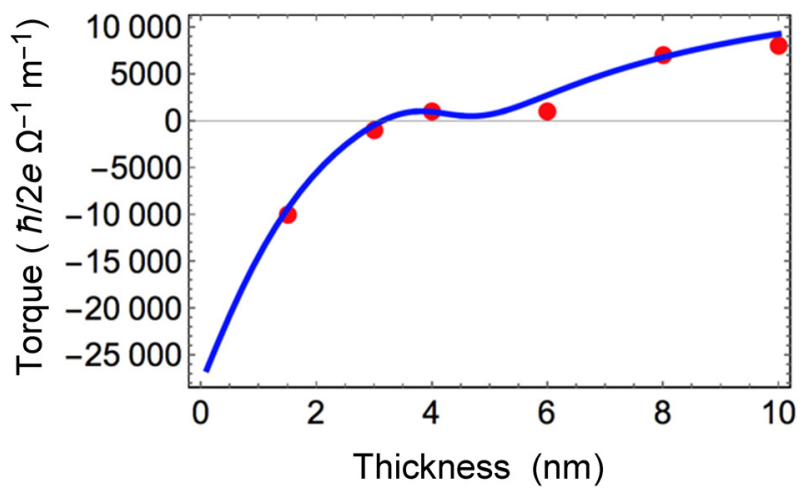

(b)

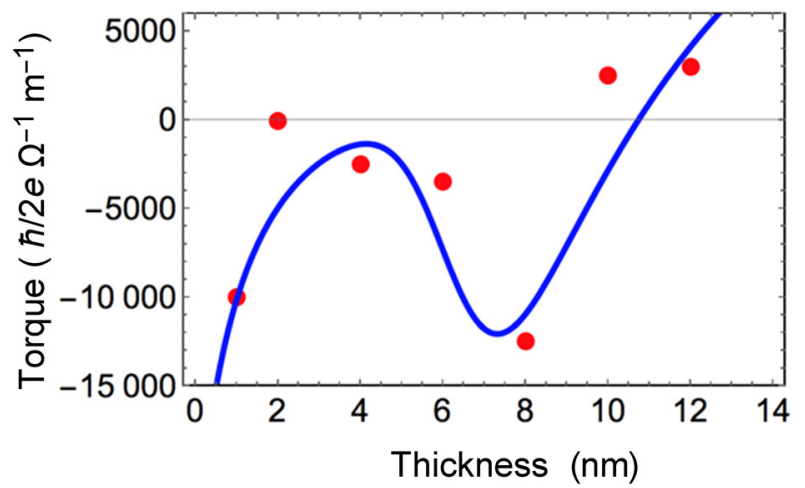

FIG. 10. Numerical fit of the experimental data of (a) Fig. 4(b) of the present work and (b) Fig. 3 from Ref. [9]. The fitting parameters are given in Table I.

diffusion lengths in the case where the magnon conductivity and spin convertance are large $\left(\sigma_{\alpha}=10^{7} \Omega^{-1} \mathrm{~m}^{-1}\right.$ and $\left.G_{\alpha}^{0}=10^{16} \Omega^{-1} \mathrm{~m}^{-2}\right)$. For small magnon diffusion lengths (green and magenta curves), the torque at large thicknesses is dominated by the SHE of the AFM layer conveyed by AFM magnons, as explained above. When increasing the magnon diffusion length (red and blue curves), the SHE from the heavy metal increases, which reduces the overall torque at large thicknesses, exhibiting the local negative maximum discussed above. For a large magnon diffusion length (black), the torque remains negative (i.e., dominated by the SHE of the heavy metal) and only reverses at very large AFM thicknesses. Comparing this figure with the experimental data of the present work [Fig. 4(b)] and Saglam et al. [9] clearly indicates that the magnon diffusion length in sputtered Fe-Mn and Ir-Mn does not exceed a few nanometers.

\section{Analysis of the experimental results}

Let us now turn to the main goal of this section and propose an interpretation of the experimental results obtained in the present experiment and in Saglam's work [9]. It appears that these two experiments represent different scenarios: in Saglam's work, a clear negative peak is observed around $6 \mathrm{~nm}$ of Fe-Mn, while in our work, a flat region appears around $4 \mathrm{~nm}$ of Ir-Mn. Phenomenologically, both behaviors can be understood based on magnon-mediated transport. The analysis we provide above suggests that in Saglam's work, the peak is associated with magnonmediated SHE from the HM layer ( $\mathrm{W}$ in their case), while in our case, the flat region implies a dominant magnon-mediated SHE from the AFM layer itself.

Using our model, we simulate both cases and attempt to fit the experimental results. To do so, we adopt the electronic properties of the HM and AFM found in the literature and adjusted the magnon parameters and interfacial conductances. The result of the fits is given in
Fig. 10. Notice that although it is possible to reproduce Saglam's result qualitatively [see Fig. 10(b)], we were not able to reproduce it quantitatively because their result is given in terms of a unitless ratio, rather than in terms of spin conductivity. For the fit produced in Fig. 10(b), we arbitrarily propose that the overall magnitude of the torque measured by Saglam is similar to ours, in the range $\pm 10^{4}(\hbar / 2 e) \Omega^{-1} \mathrm{~m}^{-1}$. Under these assumptions, the fitting we obtain reproduces the experimental trends reasonably well. We obtain a magnon diffusion length of about $6 \mathrm{~nm}$ in both cases, as well as a large magnon conductivity of about $7.7 \times 10^{6} \Omega^{-1} \mathrm{~m}^{-1}$. In our experiment, we need to assume a large electronic conductance, $G_{\text {int }}=10^{15} \Omega^{-1} \cdot \mathrm{m}^{-2}$, and an even larger spin convertance $G_{\alpha}^{0}=3 \times 10^{16} \Omega^{-1} \mathrm{~m}^{-2}$. In Saglam's experiment, we have to assume a reduced electronic conductance, $G_{\text {int }}=10^{14} \Omega^{-1} \mathrm{~m}^{-2}$, and quite a large spin convertance $G_{\alpha}^{0}=1.6 \times 10^{15} \Omega^{-1} \mathrm{~m}^{-2}$. Notice that the electron-magnon spin convertance extracted from the experimental data is one to two orders of magnitude larger than the one estimated for $\mathrm{Ir}-\mathrm{Mn}_{3}$ in Sec. III B. This difference could be attributed to a much smaller antiferromagnetic exchange energy in sputtered Ir-Mn samples (approximately $1 \mathrm{meV}$ ) compared to crystalline $\mathrm{Ir}-\mathrm{Mn}_{3}$ (approximately $40 \mathrm{meV}$ ) [53]. The present analysis of both experiments consistently suggests that interfacial electronmagnon conversion is much more efficient than interfacial electronic spin transmission in metallic AFM, while the magnon diffusion length is about ten times larger than the electronic spin relaxation length, even in a polycrystalline system.

\section{CONCLUSION}

In this work, we study the spin transport through the metallic AFM Ir-Mn by systematically varying both the temperature and the layer thickness. We measure the spin conductivity in a HM/AFM/S/FM structure via a quantitative second harmonic method. Similar to the insulating 
antiferromagnets $\mathrm{NiO}$ and $\mathrm{CoO}$ [14], the spin conductivity is also enhanced at certain thicknesses such as 1.0 and $1.5 \mathrm{~nm}$, which indicates a magnonic spin current contributes to the spin transport through the Ir-Mn layer. In addition, this magnonic spin current is expected to be enhanced around the magnetic phase transition temperature. Accordingly, an enhanced spin conductivity is observed from around 150 to $250 \mathrm{~K}$. The nonmonotonic spin conductivity dependence on the thickness of Ir-Mn arises from the interplay between three contributions: electronic spin current from Ta, magnonic spin current from Ir-Mn, and electronic spin current from Ir-Mn. To interpret this experiment, we develop a drift-diffusion model accounting for both electron and magnon transport in the heterostructure. This model allows us to discriminate between the contributions of electrons and magnons to the total spin current in Ir-Mn. We find that the electronmagnon spin convertance is one order of magnitude larger than the interfacial electronic spin conductance, while the magnon diffusion length is about ten times longer than the electronic spin relaxation length. This study demonstrates that magnonic spin transport dominates over electronic spin transport even in disordered metallic AFMs.

\section{ACKNOWLEDGMENTS}

The work reported in this publication was supported by King Abdullah University of Science and Technology (KAUST) through the Office of Sponsored Research (OSR) (Grant No. OSR-2015-CRG4-2626).

\section{APPENDIX A}

\section{Eigenmodes of a uniaxial, collinear antiferromagnet}

For simplicity, we consider the standard case of a uniaxial AFM, already treated in detail in several references (e.g., [55]) We summarize below the main results. Let us start with a bipartite AFM with $N_{s}$ spin per sublattice and described by the Hamiltonian

$$
\begin{aligned}
\hat{H}_{d d}= & -J_{F}\left(\sum_{\left\langle i, i^{\prime}\right\rangle \in A} \mathbf{S}_{i}^{A} \cdot \mathbf{S}_{i^{\prime}}^{A}+\sum_{\left\langle j, j^{\prime}\right\rangle \in B} \mathbf{S}_{j}^{B} \cdot \mathbf{S}_{j^{\prime}}^{B}\right) \\
& +\sum_{i \in A}\left[g_{A} \mu_{B} B_{\mathrm{ex}} S_{i, z}^{A}-K_{A}\left(S_{i, z}^{A}\right)^{2}\right] \\
& +\sum_{j \in B}\left[g_{B} \mu_{B} B_{\mathrm{ex}} S_{i, z}^{B}-K_{B}\left(S_{i, z}^{B}\right)^{2}\right] \\
& +J_{\mathrm{AF}} \sum_{\langle i, j\rangle \in A, B} \mathbf{S}_{i}^{A} \cdot \mathbf{S}_{j}^{B}
\end{aligned}
$$

where $A$ and $B$ refer to the different sublattices, $J_{F}$ is the ferromagnetic intrasublattice exchange, and $J_{\mathrm{AF}}$ is the antiferromagnetic intersublattice exchange. A magnetic field
$B_{\text {ex }}$ is applied along the $z$ axis, which is also the easy axis with anisotropy energy $K_{A, B}$. The brackets $\langle\ldots\rangle$ indicate when the summation is taken on the nearest neighbors only. Using Dyson-Maleev quantization, we define [55]

$$
\begin{aligned}
\mathbf{S}_{i}^{A}= & \left(S_{A}-a_{i}^{+} a_{i}\right) \mathbf{z}+\sqrt{S_{A} / 2}\left(1-\frac{1}{2 S_{A}} a_{i}^{+} a_{i}\right) a_{i} \mathbf{e}_{-} \\
& +\sqrt{S_{A} / 2} a_{i}^{+} \mathbf{e}_{+}, \\
\mathbf{S}_{j}^{B}= & -\left(S_{B}-b_{i}^{+} b_{i}\right) \mathbf{z}+\sqrt{S_{B} / 2} b_{j}^{+}\left(1-\frac{1}{2 S_{B}} b_{j}^{+} b_{j}\right) \mathbf{e}_{-} \\
& +\sqrt{S_{B} / 2} b_{j} \mathbf{e}_{+},
\end{aligned}
$$

where $a_{i}^{+}, a_{i}$ and $b_{j}^{+}, b_{j}$ are magnon creation and annihilation operators on sites $i$ and $j$, respectively, and

$$
a_{i}=\frac{1}{\sqrt{N_{s}}} \sum_{\mathbf{q}} a_{\mathbf{q}} e^{i \mathbf{q} \cdot \mathbf{r}_{i}}, b_{j}=\frac{1}{\sqrt{N_{s}}} \sum_{\mathbf{q}} b_{\mathbf{q}} e^{i \mathbf{q} \cdot \mathbf{r}_{j}}
$$

Henceforth, we obtain the following magnon Hamiltonian at the second order in magnon operators

$$
\begin{aligned}
\hat{H}_{d d} \approx & \epsilon_{0}+\sum_{\mathbf{q}}\left(\lambda_{\mathbf{q}}^{a} a_{\mathbf{q}}^{+} a_{\mathbf{q}}+\lambda_{\mathbf{q}}^{b} b_{\mathbf{q}}^{+} b_{\mathbf{q}}\right. \\
& \left.+\gamma_{\mathbf{q}} a_{\mathbf{q}} b_{-\mathbf{q}}+\gamma_{-\mathbf{q}} a_{\mathbf{q}}^{+} b_{-\mathbf{q}}^{+}\right)
\end{aligned}
$$

with

$$
\begin{aligned}
\epsilon_{0} \approx & -2 z_{n n} J_{F}\left[N_{s}\left(S_{A}^{2}+S_{B}^{2}\right)+\left(S_{A}+S_{B}\right) \sum_{\mathbf{q}} \eta_{\mathbf{q}}\right] \\
& +\mu_{B} N_{S} B\left(g_{A} S_{A}+g_{B} S_{B}\right)-N_{S}\left(K_{A} S_{A}^{2}+K_{B} S_{B}^{2}\right) \\
& -2 J_{A F} z_{n} N_{S} S_{A} S_{B}, \\
\lambda_{\mathbf{q}}^{a}= & 2 J_{A F} z_{n} S_{A}-g_{A} \mu_{B} B+K_{A}\left(2 S_{A}-1\right) \\
& +2 z_{n n} J_{F} S_{A}\left(1-\eta_{\mathbf{q}}\right), \\
\lambda_{\mathbf{q}}^{b}= & 2 J_{A F} z_{n} S_{B}+g_{B} \mu_{B} B+K_{B}\left(2 S_{B}-1\right) \\
& +2 z_{n n} J_{F} S_{B}\left(1-\eta_{\mathbf{q}}\right), \\
\gamma_{\mathbf{q}}= & 2 J_{\mathrm{AF}} z_{n} \sqrt{S_{A} S_{B}} \chi_{\mathbf{q}} .
\end{aligned}
$$

$z_{n}$ and $z_{n n}$ are the number of (antiferromagnetic) nearest neighbors and (ferromagnetic) next-nearest neighbors, respectively, and $\chi_{\mathbf{q}}=\left(1 / z_{n}\right) \sum_{\delta_{n}} e^{i \mathbf{q} \cdot \delta_{n}}, \eta_{\mathbf{q}}=\left(1 / z_{n}\right)$ $\sum_{\delta_{n n}} e^{i \mathbf{q} \cdot \delta_{n n}}$ with $\delta_{n}$ and $\delta_{n n}$ being the positions of these neighbors in the unit cell. For instance, if one takes a rock-salt magnetic unit cell with lattice parameter $a$, we 
obtain

$$
\begin{aligned}
\chi_{\mathbf{q}}= & \left(\frac{1}{3}\right)\left(\cos \frac{k_{x} a}{\sqrt{2}} \cos \frac{k_{y} a}{\sqrt{2}}+\cos \frac{k_{x} a}{\sqrt{2}} \cos \frac{k_{z} a}{\sqrt{2}}\right. \\
& \left.+\cos \frac{k_{y} a}{\sqrt{2}} \cos \frac{k_{z} a}{\sqrt{2}}\right), \\
\eta_{\mathbf{q}}= & \left(\frac{1}{3}\right)\left(\cos k_{x} a+\cos k_{y} a+\cos k_{z} a\right) .
\end{aligned}
$$

To diagonalize Eq. (A4), we use the standard Bogolyubov transformation, that is, we define

$$
a_{\mathbf{q}}=u_{\mathbf{q}} \alpha_{\mathbf{q}}+v_{\mathbf{q}} \beta_{-\mathbf{q}}^{+}, b_{-\mathbf{q}}=v_{\mathbf{q}} \alpha_{\mathbf{q}}^{+}+u_{\mathbf{q}} \beta_{-\mathbf{q}}
$$

Here, $\alpha_{\mathbf{q}}$ and $\beta_{\mathbf{q}}$ are independent bosons, such that $\left(\alpha_{\mathbf{q}}, \beta_{\mathbf{q}}\right)=0$. Under this constraint $u_{q}^{2}-v_{q}^{2}=1$, which provides

$$
\hat{H}_{d d}=\sum_{q} \hbar \omega_{q}^{\alpha} \alpha_{q}^{+} \alpha_{q}+\hbar \omega_{q}^{\beta} \beta_{q}^{+} \beta_{q}
$$

where

$$
\begin{aligned}
\hbar \omega_{q}^{\alpha} & =\frac{1}{2} \sqrt{\left(\lambda_{\mathbf{q}}^{a}+\lambda_{\mathbf{q}}^{b}\right)^{2}-\left(2 \gamma_{\mathbf{q}}\right)^{2}}+\frac{1}{2}\left(\lambda_{\mathbf{q}}^{a}-\lambda_{\mathbf{q}}^{b}\right), \\
\hbar \omega_{q}^{\beta} & =\frac{1}{2} \sqrt{\left(\lambda_{\mathbf{q}}^{a}+\lambda_{\mathbf{q}}^{b}\right)^{2}-\left(2 \gamma_{\mathbf{q}}\right)^{2}}-\frac{1}{2}\left(\lambda_{\mathbf{q}}^{a}-\lambda_{\mathbf{q}}^{b}\right), \\
u_{\boldsymbol{q}} & =\frac{1}{\sqrt{2}} \sqrt{\frac{\lambda_{\boldsymbol{q}}^{a}+\lambda_{\boldsymbol{q}}^{b}}{\sqrt{\left(\lambda_{\boldsymbol{q}}^{a}+\lambda_{\boldsymbol{q}}^{b}\right)^{2}-\left(2 \gamma_{\boldsymbol{q}}\right)^{2}}}+1} \\
v_{\boldsymbol{q}} & =-\frac{1}{\sqrt{2}} \sqrt{\frac{\lambda_{\boldsymbol{q}}^{a}+\lambda_{\boldsymbol{q}}^{b}}{\sqrt{\left(\lambda_{\boldsymbol{q}}^{a}+\lambda_{\boldsymbol{q}}^{b}\right)^{2}-\left(2 \gamma_{\boldsymbol{q}}\right)^{2}}}-1 .}
\end{aligned}
$$

By taking identical sublattices $\left(S_{A}=S_{B}, K_{A}=K_{B}\right)$ and considering nearest-neighbor interactions only $\left(J_{F}=0\right)$, this model provides the magnon dispersion, density of states, and velocity of a bcc AFM crystal,

$$
\begin{aligned}
\omega_{k} & =\mu_{B} \mu_{0} \sqrt{H_{K}\left(2 H_{E}+H_{K}\right)+H_{E}^{2}(k a)^{2} / 3}, \\
g_{m}(\omega) & =\frac{3 \sqrt{3}}{2 \pi^{2}} \frac{1}{\left(\mu_{B} \mu_{0} H_{E} a\right)^{3}} \omega \sqrt{\omega^{2}-\omega_{K}^{2}}, \\
v & =\frac{a}{\hbar \sqrt{3}} \mu_{B} \mu_{0} H_{E} \frac{\sqrt{\omega^{2}-\omega_{K}^{2}}}{\omega},
\end{aligned}
$$

where $\mu_{B} \mu_{0} H_{E}=2 J_{\mathrm{AF}} z_{n} S$ and $\mu_{B} \mu_{0} H_{K}=\omega_{K}=K(2 S$ $-1)$.

\section{Boundary conditions}

\section{a. Electron-magnon interaction}

Our aim is to derive the boundary conditions for electronic and magnonic spin currents and densities between a normal metal and a bipartite AFM metal. These conditions are governed by electron-magnon coupling at the interface. This interaction reads

$$
\hat{H}_{s d}=\sum_{i \in A} J_{s d}^{A}\left(\mathbf{r}_{i}\right) \mathbf{S}_{i}^{A} \cdot \mathbf{s}_{i}+\sum_{j \in B} J_{s d}^{B}\left(\mathbf{r}_{j}\right) \mathbf{S}_{j}^{B} \cdot \mathbf{s}_{j},
$$

where $J_{s d}^{A}\left(\mathbf{r}_{i}\right)=\sum_{l \in A_{\text {int }}} J_{s d} a_{\text {int }}^{3} \delta\left(\mathbf{r}_{i}-\mathbf{r}_{l}\right), J_{s d}^{B}\left(\mathbf{r}_{j}\right)=\sum_{l \in B_{\text {int }}}$ $J_{s d} a_{\mathrm{int}}^{3} \delta\left(\mathbf{r}_{j}-\mathbf{r}_{l}\right)$, and $\mathbf{s}_{i}=\left(1 / N_{e}\right) \sum_{\mathbf{k}, \mathbf{k}^{\prime}} c_{\mathbf{k}^{\prime}}^{+} \sigma c_{\mathbf{k}} e^{i\left(\mathbf{k}-\mathbf{k}^{\prime}\right) \cdot \mathbf{r}_{i}}$, where $N_{e}$ is the number of electrons per sublattice. As a result, the electron-magnon interaction becomes

$$
\begin{aligned}
\hat{H}_{s d}^{\|}= & \frac{1}{N_{s} N_{e}} \sum_{\mathbf{k}, \mathbf{k}^{\prime}}\left[S_{A} \tilde{J}_{\mathbf{k}^{\prime}-\mathbf{k}}^{A}+S_{B} \tilde{J}_{\mathbf{k}^{\prime}-\mathbf{k}}^{B}\right] \\
& \times\left(c_{\mathbf{k}^{\prime}, \uparrow}^{+} c_{\mathbf{k}, \uparrow}-c_{\mathbf{k}^{\prime}, \downarrow}^{+} c_{\mathbf{k}, \downarrow}\right), \\
\hat{H}_{s d}^{\perp}= & \frac{1}{\sqrt{2 N_{s}} N_{e}} \sum_{\mathbf{q}, \mathbf{k}, \mathbf{k}^{\prime}}\left[\sqrt{S_{A}} \tilde{J}_{\mathbf{k}^{\prime}-\mathbf{k}-\mathbf{q}}^{A} a_{\mathbf{q}}+\sqrt{S_{B}} \tilde{J}_{\mathbf{k}^{\prime}-\mathbf{k}-\mathbf{q}}^{B} b_{-\mathbf{q}}^{+}\right] \\
& \times c_{\mathbf{k}^{\prime}, \downarrow}^{+} c_{\mathbf{k}, \uparrow}+\text { c.c.. }
\end{aligned}
$$

Here, $N_{s}$ is the number of magnetic moments per sublattice and the Fourier transforms of the exchange constants read [47]

$$
\tilde{J}_{\mathbf{k}}^{A}=\sum_{i \in A} J_{s d}^{A}\left(\mathbf{r}_{i}\right) e^{-i \mathbf{k} \cdot \mathbf{r}_{i}}, \tilde{J}_{\mathbf{k}}^{B}=\sum_{j \in B} J_{s d}^{B}\left(\mathbf{r}_{j}\right) e^{-i \mathbf{k} \cdot \mathbf{r}_{j}} .
$$

Hamiltonian (A10) describes the interaction between the electron spin and the static order parameter. It introduces interfacial resistance, which can be accounted for through a phenomenological parameter, $G_{\text {int }}$ (see Sec. IV). Hamiltonian (A11) describes electron-magnon interactions and is responsible for conversion from electronic to magnonic spin currents at the interface. Inserting the magnon eigenmodes derived in Appendix A 1, we obtain (see also [49])

$$
\begin{aligned}
\hat{H}_{s d}^{\perp}= & \frac{1}{\sqrt{2 N_{s}} N_{e}} \sum_{\mathbf{q}, \mathbf{k}, \mathbf{k}^{\prime}}\left[\tilde{\mathcal{J}}_{\mathbf{k}^{\prime}-\mathbf{k}-\mathbf{q}}^{\alpha} \alpha_{\mathbf{q}}+\tilde{\mathcal{J}}_{\mathbf{k}^{\prime}-\mathbf{k}-\mathbf{q}}^{\beta} \beta_{\mathbf{q}}^{+}\right] \\
& \times c_{\mathbf{k}^{\prime}, \downarrow}^{+} c_{\mathbf{k}, \uparrow}+\text { c.c. },
\end{aligned}
$$

with $\quad \tilde{\mathcal{J}}_{\mathbf{k}-\mathbf{q}}^{\alpha}=u_{\mathbf{q}} \sqrt{S_{A}} \tilde{J}_{\mathbf{k}-\mathbf{q}}^{A}+v_{\mathbf{q}} \sqrt{S_{B}} \tilde{J}_{\mathbf{k}-\mathbf{q}}^{B} \quad$ and $\quad \tilde{\mathcal{J}}_{\mathbf{k}-\mathbf{q}}^{\beta}$ $=v_{\mathbf{q}} \sqrt{S_{A}} \tilde{J}_{\mathbf{k}-\mathbf{q}}^{A}+u_{\mathbf{q}} \sqrt{S_{B}} \tilde{J}_{\mathbf{k}-\mathbf{q}}^{B}$. Then, one can clearly see that mode $\alpha$ carries a spin $-\hbar$, while mode $\beta$ carries a spin $+\hbar$. Therefore, upon accumulating spin density at the interface, mode $\alpha$ is excited while mode $\beta$ is quenched. In principle, magnon-induced spin pumping should also exist in the bulk of the AFM. However, in the present theory, the AFM metal is assumed homogeneous and translationally invariant in the bulk. Therefore, at each point of the AFM metal away from the interface, magnon-induced spin pumping vanishes by symmetry. 


\section{b. Electron and magnon spin currents}

The electronic spin current generated by nonequilibrium AFM magnons $J^{s}$ and the magnon spin currents induced by nonequilibrium spin density $J^{\alpha, \beta}$ are defined [47]

$$
\begin{aligned}
J^{s} & =\frac{\mu_{B}}{A_{\text {int }}} \sqrt{N_{e}}\left\langle\partial_{t} s_{z, \mathbf{k}_{0}}\right\rangle_{\mathbf{k}_{0} \rightarrow 0} \\
& =\frac{\mu_{B}}{i \hbar A_{\text {int }}} \sum_{\mathbf{k}^{\prime \prime}}\left\langle\left[\left(c_{\mathbf{k}^{\prime \prime}+\mathbf{k}_{0}, \uparrow}^{+} c_{\mathbf{k}^{\prime \prime}, \uparrow}-c_{\mathbf{k}^{\prime \prime}+\mathbf{k}_{0}, \downarrow}^{+} c_{\mathbf{k}^{\prime \prime}, \downarrow}\right), \hat{H}_{s d}^{\perp}\right]\right\rangle, \\
J^{\alpha} & =\frac{2 \mu_{B}}{A_{\text {int }}} \sqrt{N_{s}}\left\langle\partial_{t} n_{\alpha}\right\rangle=\frac{2 \mu_{B}}{i \hbar A_{\text {int }}} \sum_{\mathbf{q}^{\prime}}\left\langle\left[\alpha_{\mathbf{q}^{\prime}}^{+} \alpha_{\mathbf{q}^{\prime}}, \hat{H}_{s d}^{\perp}\right]\right\rangle, \\
J^{\beta} & =-\frac{2 \mu_{B}}{A_{\text {int }}} \sqrt{N_{s}}\left\langle\partial_{t} n_{\beta}\right\rangle=-\frac{2 \mu_{B}}{i \hbar A_{\text {int }}} \sum_{\mathbf{q}^{\prime}}\left\langle\left[\beta_{\mathbf{q}^{\prime}}^{+} \beta_{\mathbf{q}^{\prime}}, \hat{H}_{s d}^{\perp}\right]\right\rangle .
\end{aligned}
$$

Performing the commutation, we obtain

$$
\begin{aligned}
J^{s}= & -\frac{\mu_{B}}{\hbar A_{\text {int }}} \frac{4}{\sqrt{2 N_{s}} N_{e}} \Im \\
& \times \sum_{\mathbf{q}, \mathbf{k}, \mathbf{k}^{\prime}}\left\langle\left[\tilde{\mathcal{J}}_{\mathbf{k}^{\prime}-\mathbf{k}-\mathbf{q}}^{\alpha} \alpha_{\mathbf{q}}+\tilde{\mathcal{J}}_{\mathbf{k}^{\prime}-\mathbf{k}-\mathbf{q}}^{\beta} \beta_{\mathbf{q}}^{+}\right] c_{\mathbf{k}^{\prime}, \downarrow}^{+} c_{\mathbf{k}, \uparrow}\right\rangle, \\
J^{\alpha}= & -\frac{\mu_{B}}{\hbar A_{\text {int }}} \frac{4}{\sqrt{2 N_{s}} N_{e}} \Im \sum_{\mathbf{q}, \mathbf{k}, \mathbf{k}^{\prime}}\left\langle\tilde{\mathcal{J}}_{\mathbf{k}^{\prime}-\mathbf{k}-\mathbf{q}}^{\alpha} \alpha_{\mathbf{q}} c_{\mathbf{k}^{\prime}, \downarrow}^{+} c_{\mathbf{k}, \uparrow}\right\rangle, \\
J^{\beta}= & -\frac{\mu_{B}}{\hbar A_{\text {int }}} \frac{4}{\sqrt{2 N_{s}} N_{e}} \Im \sum_{\mathbf{q}, \mathbf{k}, \mathbf{k}^{\prime}}\left\langle\tilde{\mathcal{J}}_{\mathbf{k}^{\prime}-\mathbf{k}-\mathbf{q}}^{\beta} \beta_{\mathbf{q}}^{+} c_{\mathbf{k}^{\prime}, \downarrow}^{+} c_{\boldsymbol{k}, \uparrow}\right\rangle .
\end{aligned}
$$

Notice that $J^{s}=J^{\alpha}+J^{\beta}$ because the total spin angular momentum is conserved at the interface, so the spin angular momentum leaving the electron bath must be transmitted to the magnon bath. Let us now compute these spin currents to the second order in an exchange interaction. Using the Fermi golden rule, we obtain

$$
\begin{gathered}
J^{s}=\frac{\mu_{B}}{\hbar A_{\text {int }}} \frac{2 \pi N_{\text {int }}}{N_{S} N_{e}}\left(\begin{array}{c}
\Lambda_{\alpha}^{2} \sum_{\mathbf{q}, \mathbf{k}, \mathbf{k}^{\prime}}\left[\left(1+n_{\mathbf{q}}^{\alpha}\right)\left(1-f_{\mathbf{k}, \uparrow}\right) f_{\mathbf{k}^{\prime}, \downarrow}-n_{\mathbf{q}}^{\alpha} f_{\mathbf{k}, \uparrow}\left(1-f_{\mathbf{k}^{\prime}, \downarrow}\right)\right] \delta\left(\epsilon_{\mathbf{k}}+\epsilon_{\mathbf{q}}^{\alpha}-\epsilon_{\mathbf{k}^{\prime}}\right) \\
-\Lambda_{\beta}^{2} \sum_{\mathbf{q}, \mathbf{k}, \mathbf{k}^{\prime}}\left[\left(1+n_{\mathbf{q}}^{\beta}\right) f_{\mathbf{k}, \uparrow}\left(1-f_{\mathbf{k}^{\prime}, \downarrow}\right)-n_{\mathbf{q}}^{\beta}\left(1-f_{\mathbf{k}, \uparrow}\right) f_{\mathbf{k}^{\prime}, \downarrow}\right] \delta\left(\epsilon_{\mathbf{k}}-\epsilon_{\mathbf{q}}^{\beta}-\epsilon_{\mathbf{k}^{\prime}}\right)
\end{array}\right), \\
J^{\alpha}=\frac{\mu_{B}}{\hbar A_{\text {int }}} \frac{2 \pi N_{\text {int }}}{N_{S} N_{e}} \Lambda_{\alpha}^{2} \sum_{\mathbf{q}, \mathbf{k}, \mathbf{k}^{\prime}}\left[\left(1+n_{\mathbf{q}}^{\alpha}\right)\left(1-f_{\mathbf{k}, \uparrow}\right) f_{\mathbf{k}^{\prime}, \downarrow}-n_{\mathbf{q}}^{\alpha} f_{\mathbf{k}, \uparrow}\left(1-f_{\mathbf{k}^{\prime}, \downarrow}\right)\right] \delta\left(\epsilon_{\mathbf{k}}+\epsilon_{\mathbf{q}}^{\alpha}-\epsilon_{\mathbf{k}^{\prime}}\right), \\
J^{\beta}=-\frac{\mu_{B}}{\hbar A_{\text {int }}} \frac{2 \pi N_{\text {int }}}{N_{S} N_{e}} \Lambda_{\beta}^{2} \sum_{\mathbf{q}, \mathbf{k}, \mathbf{k}^{\prime}}\left[\left(1+n_{\mathbf{q}}^{\beta}\right) f_{\mathbf{k}, \uparrow}\left(1-f_{\mathbf{k}^{\prime}, \downarrow}\right)-n_{\mathbf{q}}^{\beta}\left(1-f_{\mathbf{k}, \uparrow}\right) f_{\mathbf{k}^{\prime}, \downarrow}\right] \delta\left(\epsilon_{\mathbf{k}}-\epsilon_{\mathbf{q}}^{\beta}-\epsilon_{\mathbf{k}^{\prime}}\right) .
\end{gathered}
$$

Here, $\Lambda_{\alpha}^{2}=\left\langle\left|\tilde{\mathcal{J}}_{\mathbf{k}^{\prime}-\mathbf{k}-\mathbf{q}}^{\alpha}\right|^{2}\right\rangle \approx S_{A}\left(u_{\mathbf{q}} J_{s d}^{A}\right)^{2}+S_{B}\left(v_{\mathbf{q}} J_{s d}^{B}\right)^{2}$ and $\Lambda_{\beta}^{2}=\left\langle\left|\tilde{\mathcal{J}}_{\mathbf{k}^{\prime}-\mathbf{k}-\mathbf{q}}^{\beta}\right|^{2}\right\rangle \approx S_{A}\left(v_{\mathbf{q}} J_{s d}^{A}\right)^{2}+S_{B}\left(u_{\mathbf{q}} J_{s d}^{B}\right)^{2} \cdot N_{\text {int }}$ denotes the number of magnetic moments per sublattice at the interface. We consider diffusive interfaces such that $\mathbf{q}, \mathbf{k}, \mathbf{k}^{\prime}$ are independent [45].

We now follow Zhang and Zhang [45] and assume that the magnons are close to equilibrium such that the magnon distribution becomes $n_{\mathbf{q}}^{\alpha} \approx n_{\mathbf{q}}^{0}\left[\hbar \omega_{\mathbf{q}}^{\alpha}-\mu_{\alpha}(x)\right]$ and $n_{\mathbf{q}}^{\beta} \approx$ $n_{\mathbf{q}}^{0}\left[\hbar \omega_{\mathbf{q}}^{\beta}-\mu_{\beta}(x)\right]$, with $n_{\mathbf{q}}^{0}(\epsilon)$ being the Bose-Einstein distribution and $\mu_{\alpha, \beta}(x)$ the spatial-dependent magnon chemical potential. For electrons, we have to account for nonequilibrium contributions from both left and right leads. Indeed, while magnons are confined to the AFM layer, electrons exist on both sides of the interface. For a given population (either in the normal metal or in the antiferromagnetic metal), the nonequilibrium electron distribution reads $f_{\mathbf{k}, \sigma} \approx f_{0}\left(\epsilon_{\mathbf{k}}\right)+\partial_{\epsilon_{\mathbf{k}}} f_{0}\left(\epsilon_{\mathbf{k}}\right)\left[-\mu_{\sigma}(x)+g_{\mathbf{k}, \sigma}(x)\right]$. Here, $f_{0}\left(\epsilon_{\mathbf{k}}\right)$ is the Fermi-Dirac distribution, $\mu_{\sigma}(x)$ is the spatial-dependent chemical potential for spin $\sigma$, and $g_{\mathbf{k}, \sigma}(x)$ is the anisotropic part of the nonequilibrium distribution. Since this contribution averages out upon integration over the Fermi surface, under the summation of Eqs. (A19)-(A21), we can pose $f_{\mathbf{k}, \sigma} \approx f_{0}\left[\epsilon_{\mathbf{k}}-\mu_{\sigma}(x)\right]$. This substitution is only valid under the summation.

By expanding Eqs. (A19)-(A21) to the first order in electronic and magnonic spin-dependent chemical potentials, we obtain the boundary conditions due to the 
electron-magnon interaction

$$
\begin{aligned}
J_{\text {tot }}^{s} & =G_{\alpha}\left(\Delta \mu_{s}-\mu_{\alpha}\right)+G_{\beta}\left(\Delta \mu_{s}+\mu_{\beta}\right)+G_{\mathrm{int}} \Delta \mu_{s}, \\
J^{\alpha} & =G_{\alpha}\left(\Delta \mu_{s}-\mu_{\alpha}\right), \\
J^{\beta} & =G_{\beta}\left(\Delta \mu_{s}+\mu_{\beta}\right),
\end{aligned}
$$

where

$$
G_{\alpha, \beta}=\frac{2 \pi \mu_{B} a^{7}}{\hbar} g_{F}^{2} \Lambda_{\alpha, \beta}^{2} \int \omega\left(-\partial_{\omega} n_{0}\right) g_{m}^{\alpha, \beta}(\omega) d \omega
$$

$G_{\text {int }}$ is the interfacial conductance for electron spins that we add by hand and $\Delta \mu_{s}=\mu_{s}^{-}-\mu_{s}^{+}$is the spin-dependent chemical potential difference across the interface.

\section{Magnon diffusion}

The diffusion of magnons near equilibrium can be obtained by solving Boltzmann equation for each mode [56]

$$
v_{x} \partial_{x} n_{\mathbf{q}}^{\alpha, \beta}=-\frac{n_{\mathbf{q}}^{\alpha, \beta}-\overline{n_{\mathbf{q}}^{\alpha, \beta}}}{\tau_{m}}-\frac{n_{\mathbf{q}}^{\alpha, \beta}-n_{\mathbf{q}}^{0}}{\tau_{\text {th }}},
$$

where $n_{\mathbf{q}}^{\alpha, \beta}\left(n_{\mathbf{q}}^{0}\right)$ is the nonequilibrium (equilibrium, BoseEinstein) magnon distribution, $\overline{n_{\mathbf{q}}^{\alpha, \beta}}$ is averaged over the momentum $\mathbf{q}$, and $\tau_{m}, \tau_{\text {th }}$ are the momentum relaxation and thermalization times $\left(\tau_{m} \ll \tau_{\text {th }}\right)$, respectively. By defining $j^{\alpha, \beta}=\mp 2 \mu_{B} \int v g_{m}^{\alpha, \beta}(\omega) n_{\mathbf{q}}^{\alpha, \beta} d \omega$, one obtains the system of diffusive equations [45]

$$
\begin{aligned}
\partial_{x} j^{\alpha, \beta} & =\mp 2 \mu_{B} \frac{\mu_{\alpha, \beta}}{\tau_{t h}} \int g_{m}^{\alpha, \beta}(\omega)\left(-\partial_{\omega} n_{0}\right) d \omega, \\
j^{\alpha, \beta} & =\mp 2 \mu_{B} \sigma_{\alpha, \beta} \partial_{x} \mu_{\alpha, \beta}, \\
\frac{\mu_{\alpha, \beta}}{\left(l_{\alpha, \beta}\right)^{2}} & =\partial_{x}^{2} \mu_{\alpha, \beta},
\end{aligned}
$$

with the magnon conductivity $\sigma_{\alpha, \beta}=\left(\tau_{\mathrm{th}} / 3\right)$ $\int v^{2} g_{m}^{\alpha, \beta}(\omega)\left(-\partial_{\omega} n_{0}\right) d \omega$ and the magnon diffusion length $\left(l_{\alpha, \beta}\right)^{2}=\left[\tau_{\mathrm{th}} \sigma_{\alpha, \beta} / \int g_{m}^{\alpha, \beta}(\omega)\left(-\partial_{\omega} n_{0}\right) d \omega\right]$.

Using the dispersion relation, density of states, and magnon velocity derived in Appendix A 1, we explicitly obtain

$$
\begin{aligned}
G_{\alpha, \beta}= & S \frac{3 \sqrt{3}}{\pi}\left(\frac{J_{s d}}{\mu_{B} \mu_{0} H_{E}}\right)^{2} \frac{g_{F}^{2} \beta}{4 \hbar \mathcal{A}} \\
& \times \int_{\omega_{K}}^{k_{B} T_{N}} \omega \sqrt{\omega^{2}-\omega_{K}^{2}} \operatorname{csch}^{2}\left(\frac{\beta \omega}{2}\right) d \omega, \quad(\mathrm{A} 30) \\
\sigma_{\alpha, \beta}= & \frac{1}{\pi^{2} \sqrt{3}} \frac{\tau_{\mathrm{th}} / \hbar}{\mu_{B} \mu_{0} H_{E}} \frac{\beta}{4 \hbar a} \\
& \times \int_{\omega_{K}}^{k_{B} T_{N}} \frac{\left(\omega^{2}-\omega_{K}^{2}\right)^{3 / 2}}{\omega} \operatorname{csch}^{2}\left(\frac{\beta \omega}{2}\right) d \omega, \\
l_{\alpha, \beta}= & \frac{\tau_{t h} a}{\sqrt{3} \hbar} \mu_{B} \mu_{0} H_{E} \\
& \times \sqrt{\frac{\int_{\omega_{K}}^{k_{B} T_{N}}\left[\left(\omega^{2}-\omega_{K}^{2}\right)^{3 / 2} / \omega\right] \operatorname{csch}^{2}(\beta \omega / 2) d \omega}{\int_{\omega_{K}}^{k_{B} T_{N}} \omega \sqrt{\omega^{2}-\omega_{K}^{2}} \operatorname{csch}^{2}(\beta \omega / 2) d \omega} .}
\end{aligned}
$$

The interfacial spin convertance and bulk conductivity are given in $\Omega^{-1} m^{-2}$ and $\Omega^{-1} m^{-1}$.

[1] A. Brataas, A. D. Kent, and H. Ohno, Current-induced torques in magnetic materials, Nat. Mater. 11, 372 (2012).

[2] A. Soumyanarayanan, N. Reyren, A. Fert, and C. Panagopoulos, Emergent phenomena induced by spin-orbit coupling at surfaces and interfaces, Nature 539, 509 (2016).

[3] T. Jungwirth, J. Wunderlich, and K. Olejnik, Spin Hall effect devices, Nat. Mater. 11, 382 (2012).

[4] F. Matsukura, Y. Tokura, and H. Ohno, Control of magnetism by electric fields, Nat. Nanotechnol. 10, 209 (2015).

[5] J. Sinova, S. O. Valenzuela, J. Wunderlich, C. H. Back, and T. Jungwirth, Spin Hall effects, Rev. Mod. Phys. 87, 1213 (2015).

[6] L. Liu, C. F. Pai, Y. Li, H. W. Tseng, D. C. Ralph, and R. A. Buhrman, Spin-torque switching with the giant spin Hall effect of tantalum, Science 336, 555 (2012).

[7] Q. Shao, G. Yu, Y. W. Lan, Y. Shi, M. Y. Li, C. Zheng, X. Zhu, L. J. Li, P. K. Amiri, and K. L. Wang, Strong RashbaEdelstein effect-induced spin-orbit torques in monolayer transition metal dichalcogenide/ferromagnet bilayers, Nano Lett. 16, 7514 (2016).

[8] A. R. Mellnik, J. S. Lee, A. Richardella, J. L. Grab, P. J. Mintun, M. H. Fischer, A. Vaezi, A. Manchon, E. A. Kim, N. Samarth, and D. C. Ralph, Spin-transfer torque generated by a topological insulator, Nature 511, 449 (2014).

[9] H. Saglam, W. Zhang, M. B. Jungfleisch, J. Sklenar, J. E. Pearson, J. B. Ketterson, and A. Hoffmann, Spin transport through the metallic antiferromagnet FeMn, Phys. Rev. B 94, 140412 (2016).

[10] D. Bang, J. Yu, X. Qiu, Y. Wang, H. Awano, A. Manchon, and H. Yang, Enhancement of spin Hall effect induced 
torques for current-driven magnetic domain wall motion: Inner interface effect, Phys. Rev. B 93, 174424 (2016).

[11] L. Frangou, S. Oyarzun, S. Auffret, L. Vila, S. Gambarelli, and V. Baltz, Enhanced Spin Pumping Efficiency in Antiferromagnetic IrMn Thin Films around the Magnetic Phase Transition, Phys. Rev. Lett. 116, 077203 (2016).

[12] S. W. Jiang, S. Liu, P. Wang, Z. Z. Luan, X. D. Tao, H. F. Ding, and D. Wu, Exchange-Dominated Pure Spin Current Transport in Alq3 Molecules, Phys. Rev. Lett. 115, 086601 (2015).

[13] J. Cramer, U. Ritzmann, B.-W. Dong, S. Jaiswal, Z. Qiu, E. Saitoh, U. Nowak, and M. Kläui, Spin transport across antiferromagnets induced by the spin Seebeck effect, J. Phys. D: Appl. Phys. 51, 144004 (2018).

[14] W. Lin, K. Chen, S. Zhang, and C. L. Chien, Enhancement of Thermally Injected Spin Current through an Antiferromagnetic Insulator, Phys. Rev. Lett. 116, 186601 (2016).

[15] L. Liu, T. Moriyama, D. C. Ralph, and R. A. Buhrman, Spin-torque ferromagnetic resonance induced by the spin Hall effect, Phys. Rev. Lett. 106, 036601 (2011).

[16] H. Jaffres and A. Fert, Spin injection from a ferromagnetic metal into a semiconductor, J. Appl. Phys. 91, 8111 (2002).

[17] B. Dlubak, M.-B. Martin, C. Deranlot, B. Servet, S. Xavier, R. Mattana, M. Sprinkle, C. Berger, W. A. De Heer, F. Petroff, A. Anane, P. Seneor, and A. Fert, Highly efficient spin transport in epitaxial graphene on SiC, Nat. Phys. 8, 557 (2012).

[18] L. J. Cornelissen, J. Liu, R. A. Duine, J. B. Youssef, and B. J. van Wees, Long-distance transport of magnon spin information in a magnetic insulator at room temperature, Nat. Phys. 11, 1022 (2015).

[19] A. Manchon, I. M. Miron, T. Jungwirth, J. Sinova, J. Zelezny, A. Thiaville, K. Garello, and P. Gambardella, Current-induced spin-orbit torques in ferromagnetic and antiferromagnetic systems, arXiv:1801.09636v1.

[20] A. V. Chumak, V. I. Vasyuchka, A. A. Serga, and B. Hillebrands, Magnon spintronics, Nat. Phys. 11, 453 (2015).

[21] T. Kosub, M. Kopte, R. Huhne, P. Appel, B. Shields, P. Maletinsky, R. Hubner, M. O. Liedke, J. Fassbender, O. G. Schmidt, and D. Makarov, Purely antiferromagnetic magnetoelectric random access memory, Nat. Commun. 8, 13985 (2017).

[22] T. Kosub, M. Kopte, F. Radu, O. G. Schmidt, and D. Makarov, All-Electric Access to the Magnetic-FieldInvariant Magnetization of Antiferromagnets, Phys. Rev. Lett. 115, 097201 (2015).

[23] W. Zhang, M. B. Jungfleisch, W. Jiang, J. E. Pearson, A. Hoffmann, F. Freimuth, and Y. Mokrousov, Spin Hall Effects in Metallic Antiferromagnets, Phys. Rev. Lett. 113, 196602 (2014).

[24] T. Jungwirth, X. Marti, P. Wadley, and J. Wunderlich, Antiferromagnetic spintronics, Nat. Nanotechnol. 11, 231 (2016).

[25] V. Baltz, A. Manchon, M. Tsoi, T. Moriyama, T. Ono, and Y. Tserkovnyak, Antiferromagnetic spintronics, Rev. Mod. Phys. 90, 015005 (2018).

[26] A. V. Sadovnikov, V. A. Gubanov, S. E. Sheshukova, Y. P. Sharaevskii, and S. A. Nikitov, Spin-Wave Drop Filter Based on Asymmetric Side-Coupled Magnonic Crystals, Phys. Rev. Appl. 9, 051002 (2018).
[27] R. Lebrun, A. Ross, S. A. Bender, A. Qaiumzadeh, L. Baldrati, J. Cramer, A. Brataas, R. A. Duine, and M. Klaui, Tunable long-distance spin transport in a crystalline antiferromagnetic iron oxide, Nature 561, 222 (2018).

[28] A. V. Sadovnikov, A. A. Grachev, S. E. Sheshukova, Y. P. Sharaevskii, A. A. Serdobintsev, D. M. Mitin, and S. A. Nikitov, Magnon Straintronics: Reconfigurable Spin-Wave Routing in Strain-Controlled Bilateral Magnetic Stripes, Phys. Rev. Lett. 120, 257203 (2018).

[29] A. V. Sadovnikov, S. A. Odintsov, E. N. Beginin, S. E. Sheshukova, Y. P. Sharaevskii, and S. A. Nikitov, Toward nonlinear magnonics: Intensity-dependent spinwave switching in insulating side-coupled magnetic stripes, Phys. Rev. B 96, 144428 (2017).

[30] A. V. Sadovnikov, A. A. Grachev, E. N. Beginin, S. E. Sheshukova, Y. P. Sharaevskii, and S. A. Nikitov, Voltage-Controlled Spin-Wave Coupling in Adjacent Ferromagnetic-Ferroelectric Heterostructures, Phys. Rev. Appl. 7, 014013 (2017).

[31] A. V. Sadovnikov, E. N. Beginin, S. E. Sheshukova, Y. P. Sharaevskii, A. I. Stognij, N. N. Novitski, V. K. Sakharov, Y. V. Khivintsev, and S. A. Nikitov, Route toward semiconductor magnonics: Light-induced spin-wave nonreciprocity in a YIG/GaAs structure, Phys. Rev. B 99, 054424 (2019).

[32] S. A. Odintsov, A. V. Sadovnikov, A. A. Grachev, E. N. Beginin, Y. P. Sharaevskii, and S. A. Nikitov, Spatial-frequency selection of magnetostatic waves in a twodimensional magnonic crystal lattice, JETP Lett. 104, 563 (2017).

[33] O. Gladii, L. Frangou, G. Forestier, R. L. Seeger, S. Auffret, I. Joumard, M. Rubio-Roy, S. Gambarelli, and V. Baltz, Unraveling the influence of electronic and magnonic spincurrent injection near the magnetic ordering transition of IrMn metallic antiferromagnets, Phys. Rev. B 98, 094422 (2018).

[34] Y. Ou, D. C. Ralph, and R. A. Buhrman, Strong Enhancement of the Spin Hall Effect by Spin Fluctuations Near the Curie Point of $\mathrm{Fe}_{x} P_{1-x}$ Alloys, Phys. Rev. Lett. 120, 097203 (2018).

[35] U. H. Pi, K. Won Kim, J. Y. Bae, S. C. Lee, Y. J. Cho, K. $\mathrm{S}$. Kim, and S. Seo, Tilting of the spin orientation induced by Rashba effect in ferromagnetic metal layer, Appl. Phys. Lett. 97, 162507 (2010).

[36] Y. Wen, J. Wu, P. Li, Q. Zhang, Y. Zhao, A. Manchon, J. Q. Xiao, and X. Zhang, Temperature dependence of spin-orbit torques in Cu-Au alloys, Phys. Rev. B 95, 104403 (2017).

[37] C. O. Avci, K. Garello, M. Gabureac, A. Ghosh, A. Fuhrer, S. F. Alvarado, and P. Gambardella, Interplay of spin-orbit torque and thermoelectric effects in ferromagnet/normalmetal bilayers, Phys. Rev. B 90, 224427 (2014).

[38] J. Kim, J. Sinha, S. Mitani, M. Hayashi, S. Takahashi, S. Maekawa, M. Yamanouchi, and H. Ohno, Anomalous temperature dependence of current-induced torques in $\mathrm{CoFeB} / \mathrm{MgO}$ heterostructures with Ta-based underlayers, Phys. Rev. B 89, 174424 (2014).

[39] X. Qiu, P. Deorani, K. Narayanapillai, K. S. Lee, K. J. Lee, H. W. Lee, and H. Yang, Angular and temperature dependence of current induced spin-orbit effective fields in $\mathrm{Ta} / \mathrm{CoFeB} / \mathrm{MgO}$ nanowires, Sci. Rep. 4, 4491 (2014). 
[40] Y. Ou, C.-F. Pai, S. Shi, D. C. Ralph, and R. A. Buhrman, Origin of fieldlike spin-orbit torques in heavy metal/ferromagnet/oxide thin film heterostructures, Phys. Rev. B 94, 140414 (2016).

[41] Q. Hao and G. Xiao, Giant spin Hall effect and magnetotransport in a $\mathrm{Ta} / \mathrm{CoFeB} / \mathrm{MgO}$ layered structure: $\mathrm{A}$ temperature dependence study, Phys. Rev. B 91, 224413 (2015).

[42] T. Ambrose and C. L. Chien, Finite-Size Effects and Uncompensated Magnetization in Thin Antiferromagnetic CoO Layers, Phys. Rev. Lett. 76, 1743 (1996).

[43] P. Van der Zaag, Y. Ijiri, J. Borchers, L. Feiner, R. Wolf, J. Gaines, R. Erwin, and M. Verheijen, Difference Between Blocking and Néel Temperatures in the Exchange Biased $\mathrm{Fe}_{3} \mathrm{O}_{4} / \mathrm{CoO}$ System, Phys. Rev. Lett. 84, 6102 (2000).

[44] A. Kohn, A. Kovacs, R. Fan, G. J. McIntyre, R. C. Ward, and J. P. Goff, The antiferromagnetic structures of $\mathrm{IrMn}_{3}$ and their influence on exchange-bias, Sci. Rep. 3, 2412 (2013).

[45] S. S. L. Zhang and S. Zhang, Spin convertance at magnetic interfaces, Phys. Rev. B 86, 214424 (2012).

[46] Y. Cheng, K. Chen, and S. Zhang, Interplay of magnon and electron currents in magnetic heterostructure, Phys. Rev. B 96, 024449 (2017).

[47] H. Adachi, K. Uchida, E. Saitoh, and S. Maekawa, Theory of the spin Seebeck effect, Rep. Prog. Phys. 76, 036501 (2013).

[48] S. A. Bender, R. A. Duine, and Y. Tserkovnyak, Electronic Pumping of Quasiequilibrium Bose-Einstein-
Condensed Magnons, Phys. Rev. Lett. 108, 246601 (2012).

[49] Y. Ohnuma, H. Adachi, E. Saitoh, and S. Maekawa, Spin Seebeck effect in antiferromagnets and compensated ferrimagnets, Phys. Rev. B 87, 014423 (2013).

[50] S. M. Rezende, R. L. Rodriguez-Suarez, and A. Azevedo, Diffusive magnonic spin transport in antiferromagnetic insulators, Phys. Rev. B 93, 054412 (2016).

[51] S. Takei and M. Mohseni, Quantum control of topological defects in magnetic systems, Phys. Rev. B 97, 064401 (2018).

[52] J. Bass and W. P. Pratt, Spin-diffusion lengths in metals and alloys, and spin-flipping at metal/metal interfaces: An experimentalist's critical review, J. Phys.-Condens. Mat. 19, 183201 (2007).

[53] L. Szunyogh, B. Lazarovits, L. Udvardi, J. Jackson, and U. Nowak, Giant magnetic anisotropy of the bulk antiferromagnets IrMn and $\mathrm{IrMn}_{3}$ from first principles, Phys. Rev. B 79, 020403 (2009).

[54] Y. Xu, S. Wang, and K. Xia, Spin-Transfer Torques in Antiferromagnetic Metals from First Principles, Phys. Rev. Lett. 100, 226602 (2008).

[55] A. B. Harris, D. Kumar, B. I. Halperin, and P. C. Hohenber, Dynamics of an antiferromagnet at low temperatures - spin-wave damping and hydrodynamics, Phys. Rev. B 3, 961 (1971).

[56] S. S. Zhang and S. Zhang, Magnon Mediated Electric Current Drag Across a Ferromagnetic Insulator Layer, Phys. Rev. Lett. 109, 096603 (2012). 